\title{
ORDINAL LOGISTIC REGRESSION ANALYSIS OF RFID DOORWAY PORTAL PERFORMANCE AS A FUNCTION OF SYSTEM DESIGN PARAMETERS
}

\author{
A Thesis \\ presented to \\ the Faculty of California Polytechnic State University, \\ San Luis Obispo
}

\author{
In Partial Fulfillment \\ of the Requirements for the Degree \\ Master of Science in Industrial Engineering
}

by

Anton Slobodnik

April 2010 
(C) 2010

Anton Slobodnik

ALL RIGHTS RESERVED 


\section{COMMITTEE MEMBERSHIP}

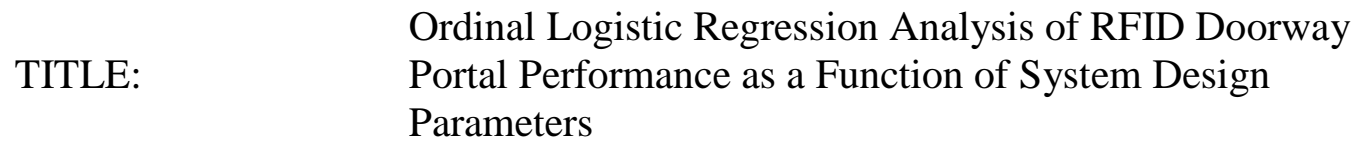

AUTHOR: Anton Slobodnik

DATE

SUBMITTED: April 1, 2010

Committee Chair Dr. Tali Freed, Ph.D.

Committee Member Dr. Jose Macedo, Ph.D.

Committee Member Dr. Lizabeth Schlemer, Ph.D. 


\begin{abstract}
Ordinal Logistic Regression Analysis of RFID Doorway Portal Performance as a Function of System Design Parameters

by

Anton Slobodnik

This research effort examined the read rate differences in a passive ultra high frequency (UHF) Radio Frequency Identification (RFID) doorway portal as a function of antenna placement, doorway size and composition, reader manufacturer, tag type, and tag orientation.

In this analysis of a RFID system, the response observations were modeled under an ordinal logistic regression model. The selection of a categorical analysis method was due to the flaws associated with the initial design of the experiment, which resulted in high valued observations which would have skewed the results of a quantitative model.

The ordinal regression analysis of the data indicates that out of the parameters studied, system performance is increased when a Sirit reader system, setup in a double doorway, with an antenna at the top of the door frame as well as the sides are used in conjunction with Sirit NXP tags.
\end{abstract}

Keywords: RFID, passive, UHF, RFID portal, RFID system design 


\section{TABLE OF CONTENTS}

LIST OF TABLES ............................................................................ viii

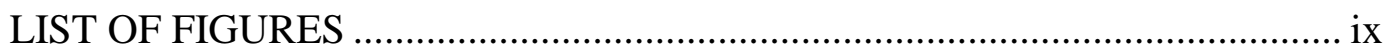

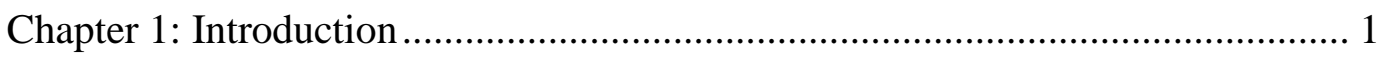

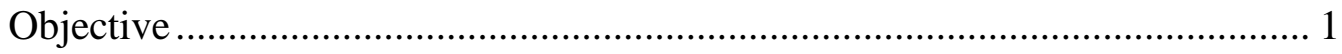

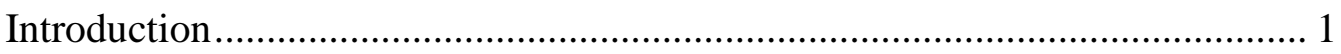

Chapter 2: Literature Review .................................................................. 3

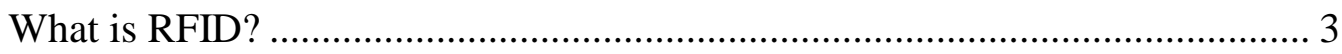

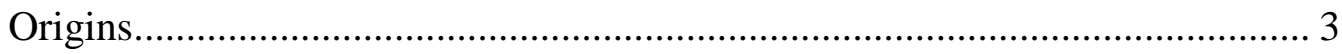

Basic Systems .................................................................................... 4

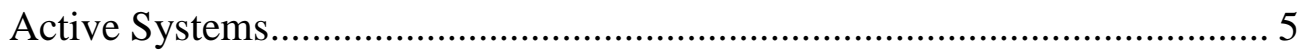

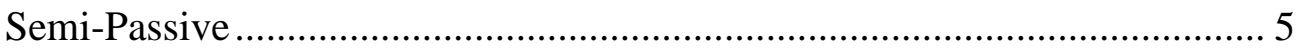

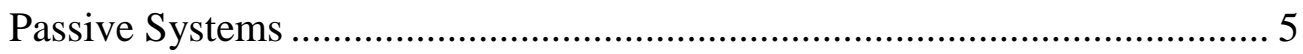

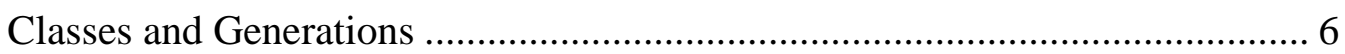

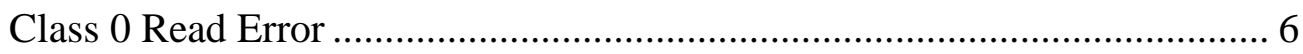

Air Interference Protocol ..................................................................... 7

Electromagnetic Wave Propagation............................................................... 7

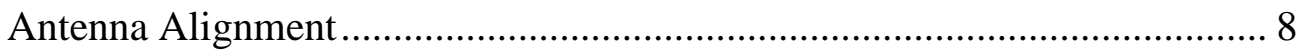

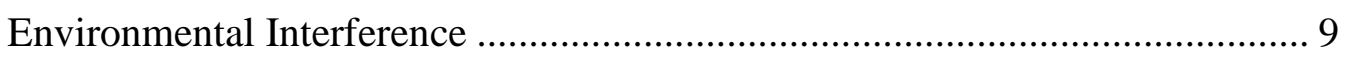

Ambient Electromagnetic Noise .......................................................... 9

Effects of Metal and Liquid ................................................................. 10

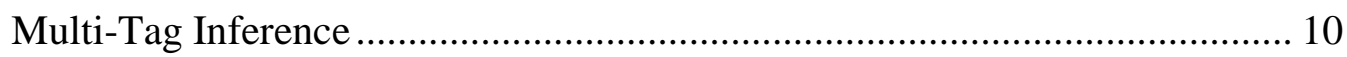

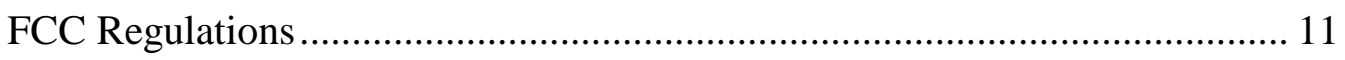

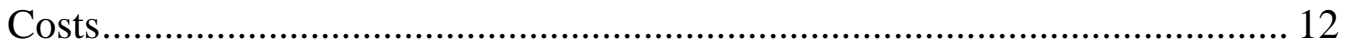

Communication Channel Sensitivity ..................................................... 12

Benchmarking an RFID system ......................................................... 13

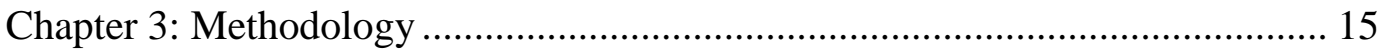

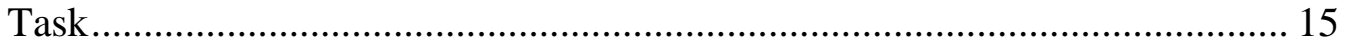

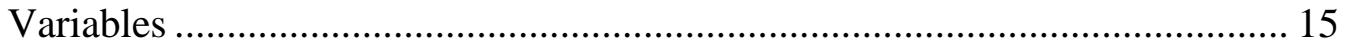

Independent (Experimental) Variables .................................................. 15

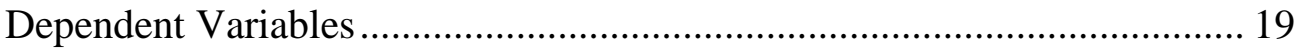

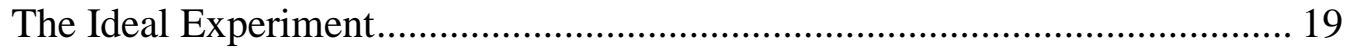

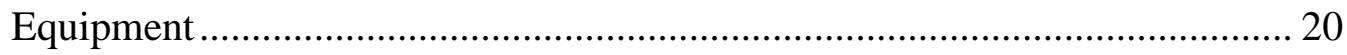

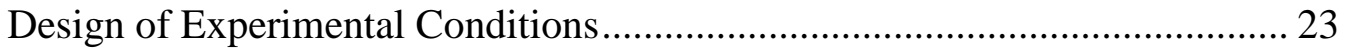




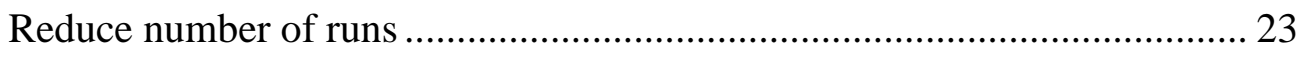

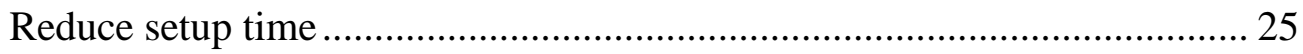

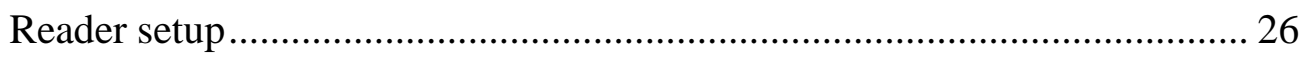

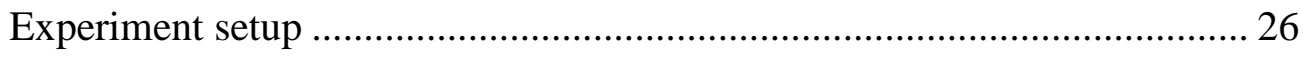

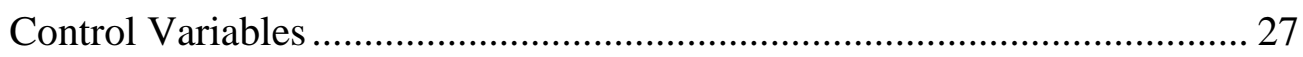

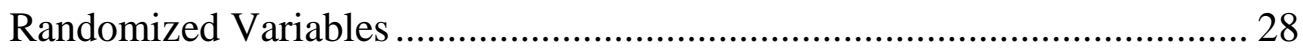

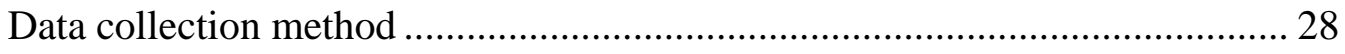

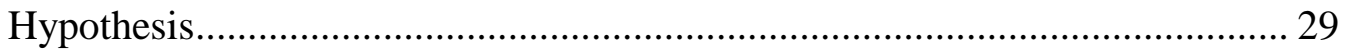

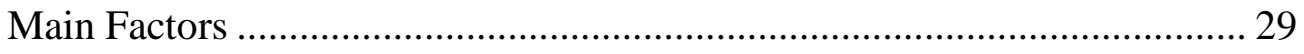

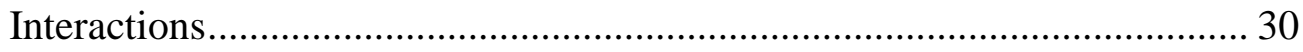

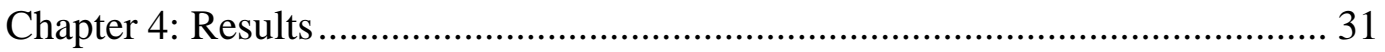

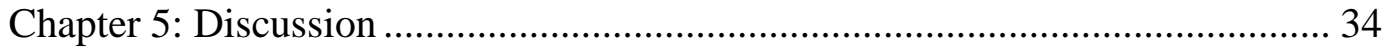

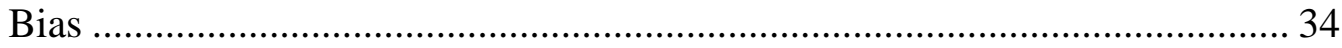

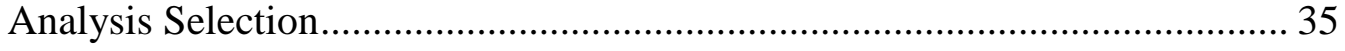

Poisson Regression …………………………………………………. 35

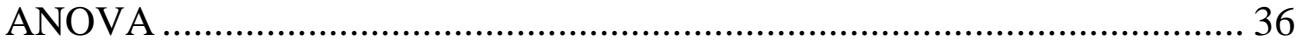

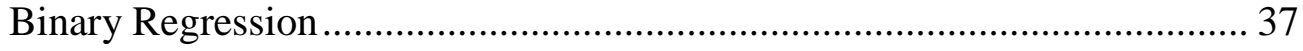

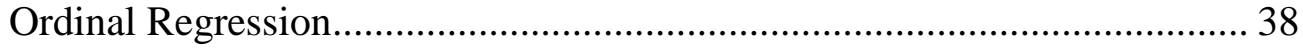

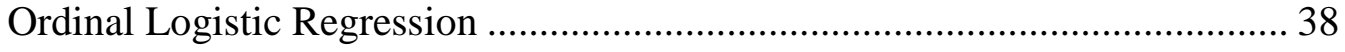

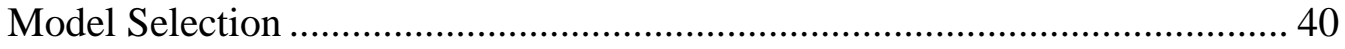

Definition of the Ordinal Response .............................................................. 40

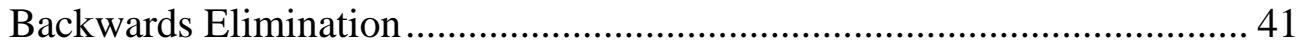

Significance of the Day (Run Order) ............................................................ 41

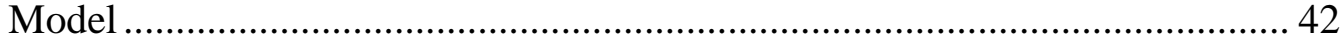

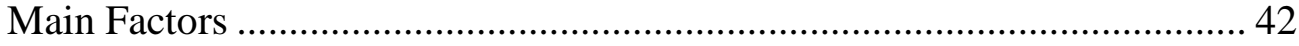

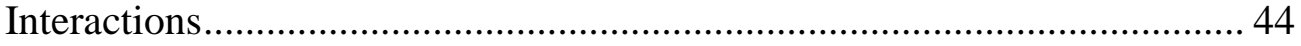

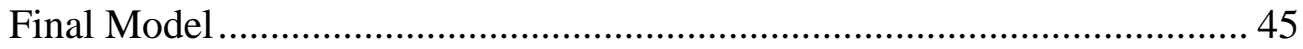

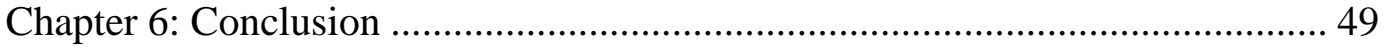

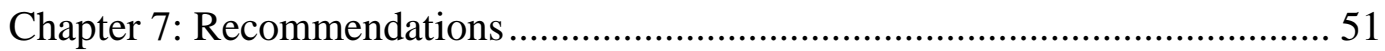

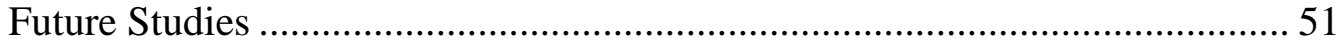

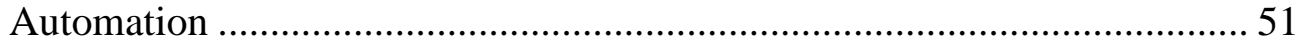

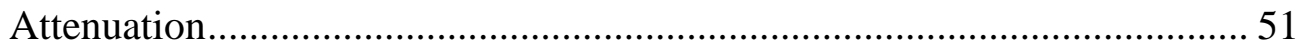

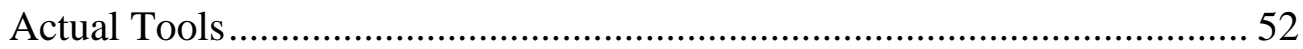

Temperature and Humidity ........................................................................ 52 


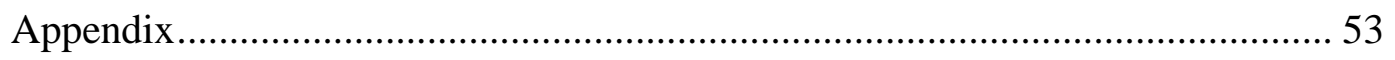

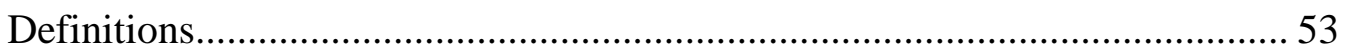

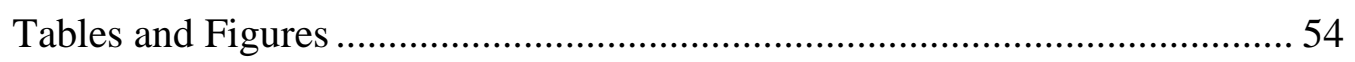

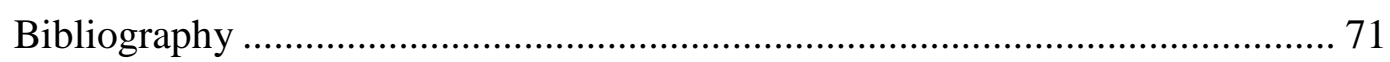




\section{LIST OF TABLES}

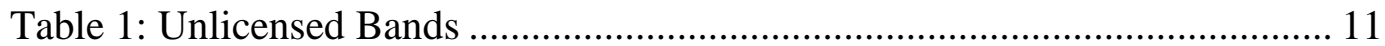

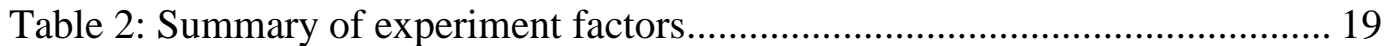

Table 3: Setup difficulty ................................................................................ 25

Table 4: Basic Descriptive statistics of Read Count ........................................... 31

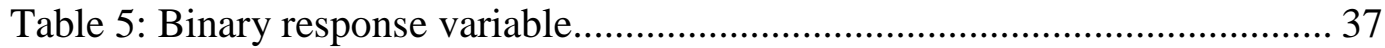

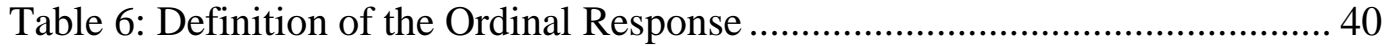

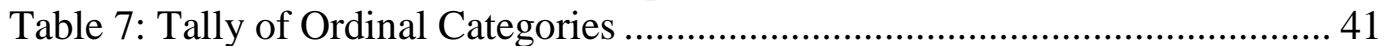

Table 8: SAS Type 3 Analyses of Effects ............................................................ 45

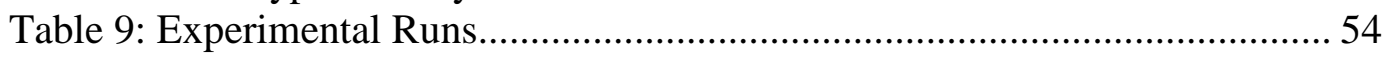

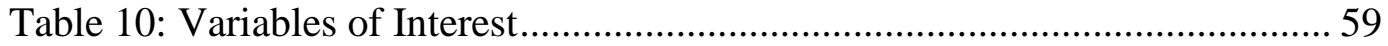

Table 11: Ordinal Logistic Regression output Minitab (no interactions) ............. 60

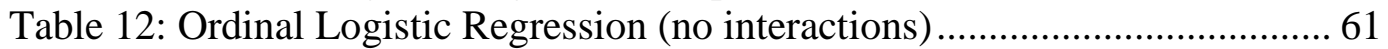

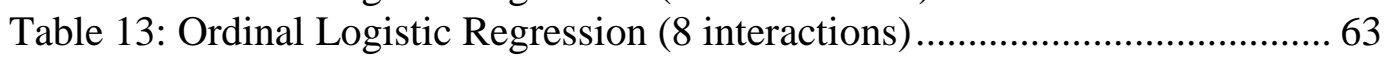

Table 14: Ordinal Logistic Regression (Final Model) ......................................... 66 


\section{LIST OF FIGURES}

Figure 1: Electromagnetic field propagation (Dobkin, RFID Basics: Antenna

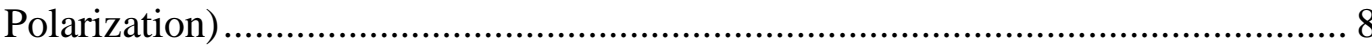

Figure 2: Circularly polarized antenna (Dobkin, RFID Basics: Antenna

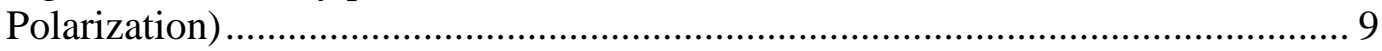

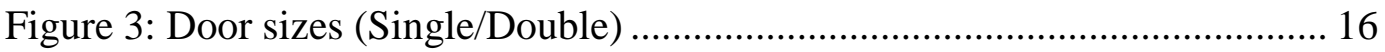

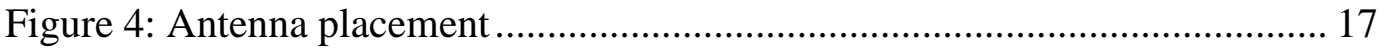

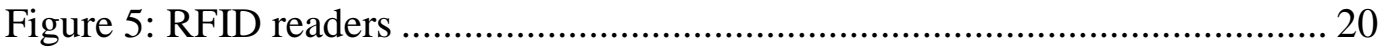

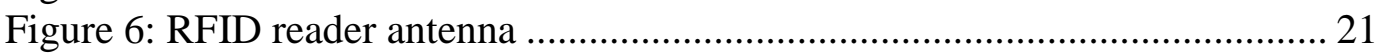

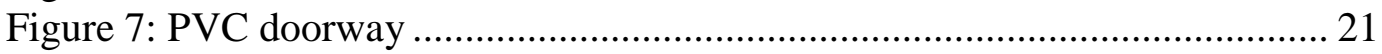

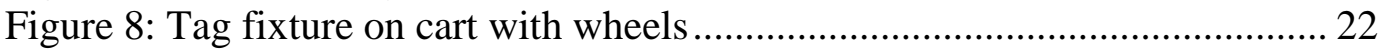

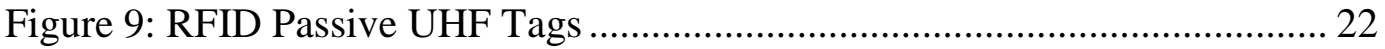

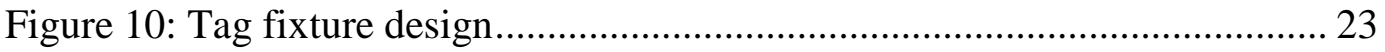

Figure 11: Tag placement on foam board ..................................................... 24

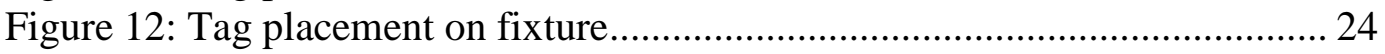

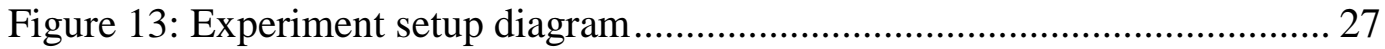

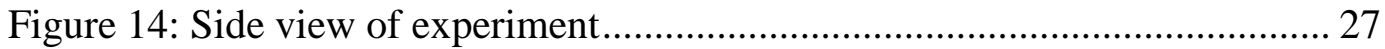

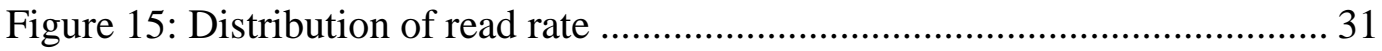

Figure 16: Main Effect Plot for read counts over 300 ..................................... 32

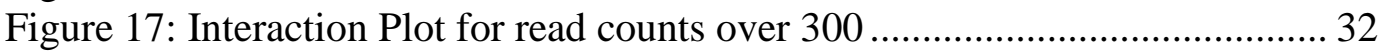

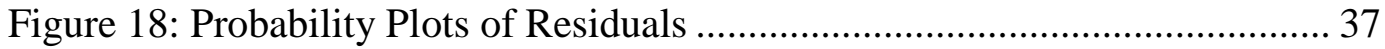

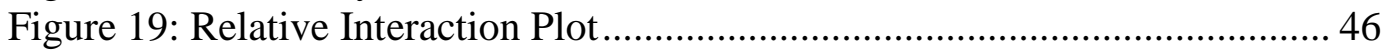

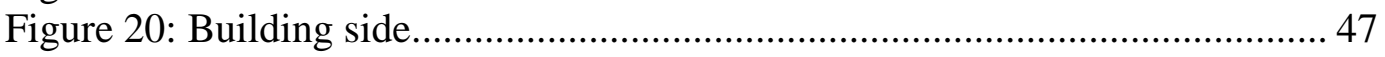

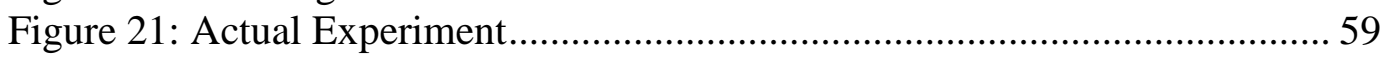

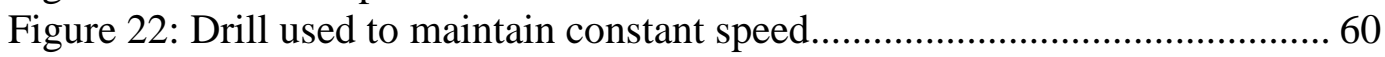

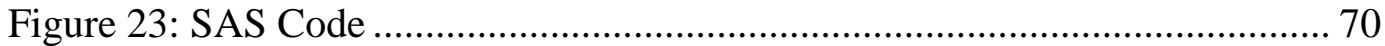




\section{Chapter 1: Introduction}

\section{Objective}

In this research effort, a design of experiments approach was used to evaluate RFID tag - reader communication performance based on portal configuration designed for industrial doorways. The study examined the read rate differences in a passive ultra high frequency (UHF) Radio Frequency Identification (RFID) doorway portal as a function of antenna placement, doorway size and composition, reader manufacturer, tag type, tag location, and tag orientation.

\section{Introduction}

RFID technology presents opportunities for many entities to reduce costs and improve efficiency by integrating this new technology within their businesses. Potential prospects for RFID implementation are equipment or tool rooms with other applications where movement of inventory items should be reported. In this case RFID technology will provide real time data, detailing the location of equipment or who is using the tool. This information will greatly increase equipment utilization and prevent their misplacement, with potential to significantly reduce costs.

The nature of such a scenario will require the implementation of RFID technology in a doorway, with tools traveling through the portal at random orientations. Due to the physics behind electromagnetic field propagation, orientation of reader antenna relative to tag antenna is an extremely important 
factor in system performance. With no means of controlling tag orientation, the selection, placement, and orientation of reader antennas becomes crucial to the creation of a capable RFID doorway system.

The experiment is conducted to find the best location(s) and orientation for reader antenna(s) in a doorway - single door and double doors - that will maximize system performance regardless of tag orientation within the portal. Performance of reader brand and tag type was also evaluated as part of the study. 


\section{Chapter 2: Literature Review}

\section{What is RFID?}

Radio Frequency Identification (RFID) is a relatively new technology which holds promise to replace other forms of identification, such as the bar code (Sweeney). RFID offers an advantage over other technologies because it does not require a line of sight between the reader and object, and is capable of scanning multiple objects within a fraction of a second. With proper design and integration, RFID technology can provide real-time in-transit visibility (ITV) and give an entire system view of a company's supply chain.

The basics of RFID technology involves a transponder communicating with a reader at which point the reader records the transponder's unique identification number or string. Radio waves are used as a channel of communication between the two, with slight variations in technology depending on the frequency used.

\section{Origins}

One of the very first uses of RFID technology was in World War II. The Allies would use an early version of the technology to determine whether or not planes flying overhead were friend or foe. Since the time of World War II, RFID has gained widespread use and support.

In 1999, several personnel at the Massachusetts Institute of Technology (MIT), with help from a consortium of companies, formed the Auto-ID Center: a research center that would further develop the nature and use of RFID technology. 
In order to help unleash the full potential of RFID, an electronic standard was developed. The Electronic Product Code (EPC) is essentially an electronic version (with a few differences) of the Universal Product Code (UPC) found on bar codes. In November of 2003, the Auto-ID Center at MIT turned over its work to EPCglobal Inc. which is the main entity pushing for and developing a global standard for RFID technology. Today, most companies have standardized on one protocol, with near all RFID systems being developed under the EPC Generation 2.0 protocol.

The success and spread of RFID technology can be largely attributed to the mandates put forth by Wal-Mart and the U.S. Department of Defense (DoD) which have been strong supporters of RFID implementation. In June of 2003, Linda Dillman, Chief Information Officer (CIO) of Wal-Mart, announced that Wal-Mart will be issuing a mandate to its suppliers to put RFID tags on every case and pallet that entered a Wal-Mart distribution center. In October of 2003, Michael Wynne, acting Under Secretary of Defense for Acquisition, Technology, and Logistics, released a policy paper detailing a passive RFID program for all 40,000 DoD suppliers. Needless to say the industry mandates have been a potent force in RFID technology spread and development.

\section{Basic Systems}

An RFID solution consists of three parts: a transponder or tag, a reader with an antenna, and a host computer to process the information. Most of the criteria for RFID systems depend on the type of tag used. Tags can be active, passive or semi-passive. 


\section{Active Systems}

An active tag is a transponder with its own power source (battery). The power from the battery is used to run the chip's circuitry and broadcast the signal to the reader. Active tags are capable of communicating with readers at great ranges with some of the more powerful tags capable of being read from a kilometer away (Sweeney).

\section{Semi-Passive}

A semi-passive tag is a transponder with its own power source just like an active tag, but the power from the battery is used to only run the onboard circuitry. To broadcast the signal the tag derives power from the electromagnetic field created by the signal from the RFID reader. These tags are generally used to monitor sensor inputs, such as temperature or movement without being within the interrogation zone of a reader.

\section{Passive Systems}

A passive tag is a transponder that does not require a battery to run. The power to run the chip's circuitry and to communicate with a reader is derived from the electromagnetic field emitted by the reader. Passive tags are also the cheapest variant of RFID technology with a price of about ten cents per unit. Because of the relatively cheap price, passive systems have been the requirements imposed by mandates in various industries.

For passive systems, the choice of operating frequency is very important. RFID frequencies are generally classified into three main group divisions: Low 
Frequency, High Frequency, and Ultra High Frequency. System operating range will generally increase as the frequency is increased.

Frequency is also a determinant of how the tag will power up and communicate with the reader. The region closest to the source of an electrical current is called the induction field. Outside the induction field is the radiation field. In low and high frequency technology, tag - reader interaction is done in the induction field, while ultra high frequencies use the radiation field.

\section{Classes and Generations}

Tags can also come in a variety of classes and generations. The Auto-ID center has developed a layered class structure to describe the technology and capabilities of RFID systems (Sarma and Engels). The main difference between class 0 and class I tags is the ability to modify data. Class 0 tags are read only and use identification number that the manufacturer writes to the tag. Class 1 tags are $\mathrm{read} / \mathrm{write}$ tags, meaning that a tag can be programmed with any identification number by the user. Class II tags are passive tags with additional functionality such as memory or encryption. Class III tags are semi-passive RFID tags, and class IV are active tags. The generation refers to the standard employed by the technology. Generation 2 protocol is currently the newest and most accepted standard.

\section{Class 0 Read Error}

Class 0 systems are susceptible to erroneous read of tags. It has been found through experiment that class 0 system can potentially register a successful read of a tag that is nowhere in the system (Ramakrishnan and Deavours). A 
"ghost tag" was registered by the system $0.1 \%$ of the time, and this unwanted

effect appears to be limited to just the class 0 technology.

\section{Air Interference Protocol}

Air interference protocols dictate how the readers and tags talk to each other and how data is kept from colliding. As RFID systems grow and increase in size, the role of an air interference protocol becomes very important. Situations when multiple tags attempt to communicate at the same time may lead to a collision which may result in a null, with no data received by the reader. The protocol uses various algorithms to time the responses and reduce the chance of a collision. It is important to note that number of tags present in an interrogation field may reduce the performance of an RFID system depending on the situation. For example if 1 tag is interrogated by the reader for 30 seconds, expected results will yield $\sim 1000$ reads. If 10 tags are interrogated by the reader for 30 seconds, expected results will yield 100 reads (Sweeney).

\section{Electromagnetic Wave Propagation}

Electromagnetic waves propagate through the vacuum or matter in form of two components; the electric and magnetic fields. The components oscillate in phase perpendicular to each other and perpendicular to the direction of energy propagation. A relationship exists between the two components where one causes the other and vice versa. In order to power up the transponder, the propagating waves emitted from the reader antenna must be directed along the linear antenna axis (Dobkin, RFID Basics: Antenna Polarization). 


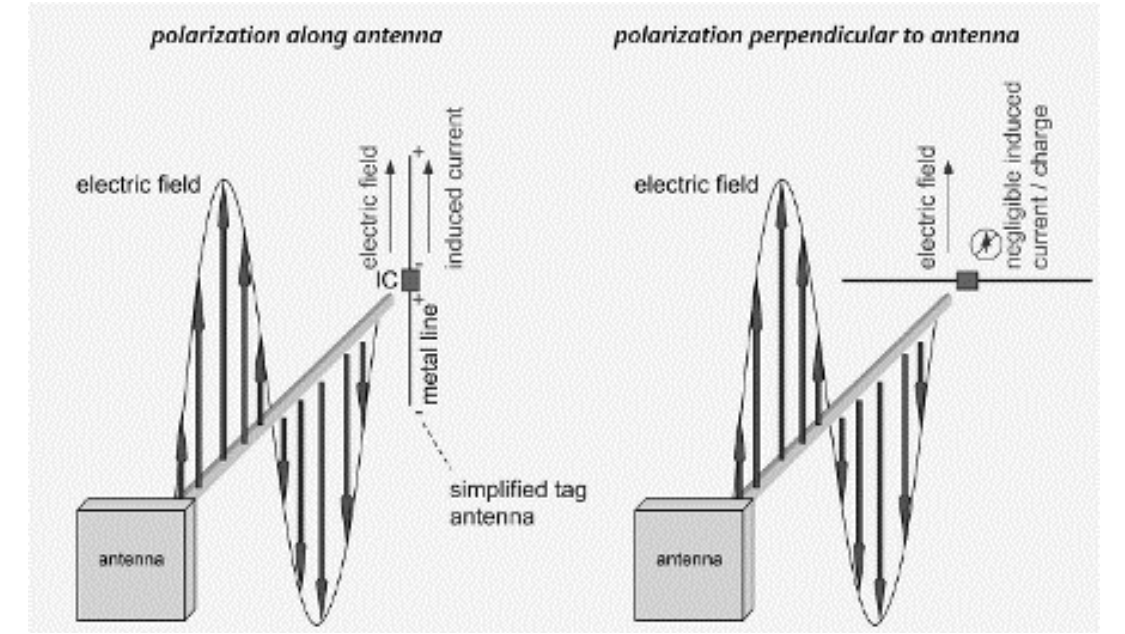

Figure 1: Electromagnetic field propagation (Dobkin, RFID Basics: Antenna Polarization)

If the propagating electric field is directed perpendicular to the tag antenna axis, the result will produce negligible current, no detectable voltage, and thus no power in the transponder.

\section{Antenna Alignment}

Antenna polarization or the orientation of the electric field of the propagating electromagnetic wave emitted from the antenna is an important factor for RFID systems. As explained above, if the electric field and antenna axis are perpendicular to each other, the RFID communication between the tag and reader will not work. This is true for linearly polarized antennas. In order to increase readability of tags in any orientation, circularly polarized antennas are used. In this case, the orientation of the electric field is time-dependent. Thus, a circularly polarized wave will interact with a tag antenna tilted at any angle, but in every case the transmitted power that is retrieved by the tag will be less than that of a linearly polarized system (Dobkin, RFID Basics: Antenna Polarization). 


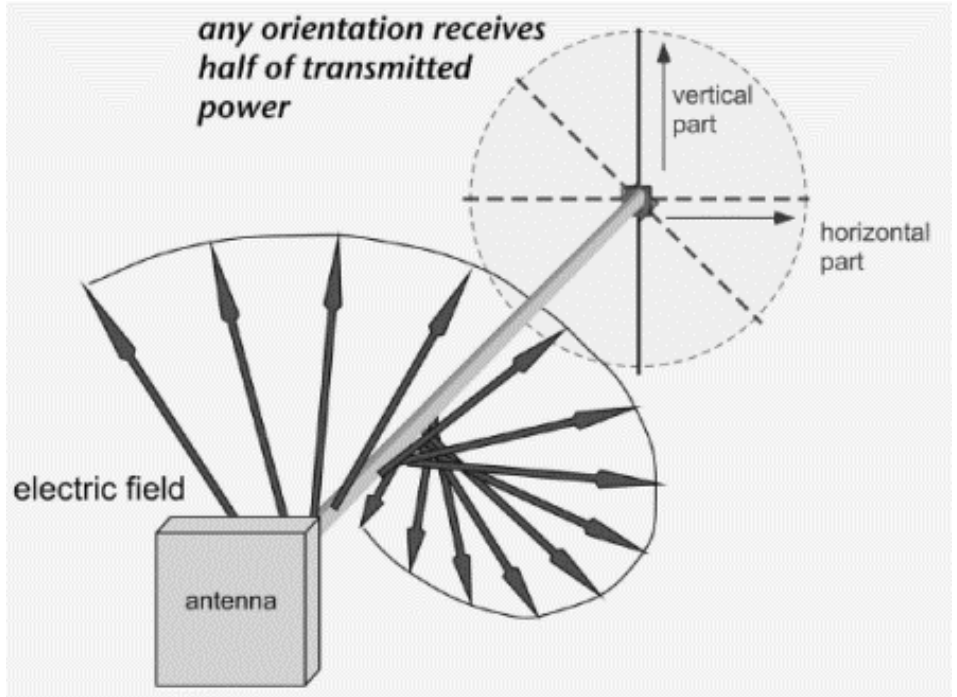

Figure 2: Circularly polarized antenna (Dobkin, RFID Basics: Antenna Polarization)

\section{Environmental Interference}

There is no silver bullet when it comes to setting up an RFID system. Each application is very unique and is heavily dependent on the environment. There are several environmental factors that need to be considered, such as Ambient Electromagnetic Noise (AEN) and the presence of metals and liquids near or in the RFID system.

\section{Ambient Electromagnetic Noise}

Any equipment that emits radio waves close to the frequency of the RFID technology may have negative effects on the performance of the system. Interference can propagate from other RFID systems or other technology present in the area of the proposed RFID implementation. It is also possible for the interference to originate from neighboring sites. In order to setup a successful RFID system, a proper evaluation of AEN is recommended by using a spectrum analyzer and looking at various frequencies propagating throughout the site (Sweeney). 


\section{Effects of Metal and Liquid}

As a general rule, metals tend to reflect electromagnetic waves and liquids absorb them. This is an important factor to understand when tagging objects for an RFID system and has been an area of much research. Conductors (metals) and dielectric materials (liquid) can drastically effect or detune the properties of a tag antenna and greatly reduce system performance. If the product consists of metals it may lead to destructive or constructive interference as the electromagnetic waves are reflected. The constructive interference can potential improve read range of the tags, when metal is placed $4-8 \mathrm{~cm}$ on the other side of the tag away from the reader (Dobkin and Weigand, Evironmental Effects on RFID Tag Antennas). In order to setup a successful system, the communication between tag and reader should not be obstructed by either water or metal. The effects of such environmental elopements have been shown to significantly degrade system performance (Aroor and Deavours).

\section{Multi-Tag Inference}

Another important factor for developing large RFID systems with multiple transponders in the vicinity of a reader is the distance relation of tags to each other. When tags are in close proximity to one another, an unwanted destructive interference may occur, and decrease the ability of the tags to be read (Weigand and Dobkin). Such effects are of practical importance for densely packed arrays of tagged items. 


\section{FCC Regulations}

Because the radio frequency waves have such a big impact on other receivers and are relatively difficult to block, various government agencies have imposed tight regulation on the bands. The Federal Communications Commission (FCC) and its equivalent in many other countries have developed spectrum licensing which specifies the frequencies, communication means, amplitude, and uses for various frequencies. The FCC has established licensed and unlicensed bands for use by industry and people.

Licensed bands are frequencies that cost money to use. In the 1980's and 90's licensed frequency bands were an area of much speculations which has resulted in many spectacular overnight millionaires. The unlicensed bands allow anyone to use these frequencies as long as people follow certain rules of transmission and broadcast. In the United States, the FCC has allocated the following LF, HF, and UHF bands for unlicensed use:

Table 1: Unlicensed Bands

\begin{tabular}{|c|c|} 
Table 1: Unlicensed Bands \\
\begin{tabular}{|c|c|}
\hline \multicolumn{2}{|c|}{ Unlicensed Bands } \\
\hline Frequency & Band \\
\hline Low & $125-134 \mathrm{kHz}$ \\
High & $13.56 \mathrm{MHz}$ \\
Ultra High & $902-928 \mathrm{MHz}$ \\
\hline
\end{tabular}
\end{tabular}

Even though the bands are unlicensed, there still are rules to follow. For example: the FCC puts a limit on the maximum amount of power that an antenna is allowed to output. 


\section{Costs}

The cost of an RFID system can be divided into three categories;

transponder, reader, and middleware and server. As of 2005, the cost of a passive RFID tag ranged from 20 cents (simple, high volume tags) to several dollars (transponder embedded in a key fob). Active tags on the other hand range from ten to over fifty dollars per tag (RFID System Components and Costs). Middleware and servers needed to run the system may cost anywhere from a few, to tens of thousands of dollars depending on the complexity of the system.

In past half decade the price of passive RFID tags have dropped from 20 cents to roughly 10 cents. Readers can range in price from a few hundred to a few thousand dollars depending on the technology needs of the system. Additionally, Forester Research estimated that it would cost a 12 billion dollar consumer product manufacturer over $\$ 100,000$ for consulting and integration, over $\$ 300,000$ for the time of the internal project team, and $\$ 80,000$ for tag and reader testing (RFID System Components and Costs). All these costs are added on top of the hardware costs and accumulate to a substantial investment. Nonetheless, companies are integrating RFID technology in their business flow and showing that a return on investment can be achieved.

\section{Communication Channel Sensitivity}

Passive RFID system performance depends on two channels of communication that need to occur successfully in order for the system to function properly. The forward channel of communication (reader-to-tag) must occur in which the tag will be able to harness enough energy from the reader. Once the tag 
has powered-on it will transmit information back to the reader (tag-to-reader), which is referred to as the reverse channel communication.

Conventional wisdom that has been widely accepted states that the limiting factor in a RFID system is the forward channel of communication. Implying that if a tag gets enough power to function the system will communicate successfully. New evidence seems to suggest that this is not the case and under certain conditions the reverse channel may in fact be a limiting factor in the system (Aroor and Deavours), with strong indications that the tag-reader communication is reverse-link limited.

\section{Benchmarking an RFID system}

In order to benchmark RFID systems and compare performance various metrics are used. Some of these measurements are read distance, attenuationthresholding, whether the tag was read, time to first read, or the number of reads per unit time.

The read range of a system is one of the more common attributes and is usually one of the first questions posed. For this metric, the "successful" maximum distance between the tag and reader is recorded. Such measurements are often used to create a radar chart of the range and shape of the passive RFID communication area. Attenuation-thresholding was proposed in 2007 (Hodges, Thorne and Mallinson) by increasing the attenuation under a computer control until the tag read-rate drops below a chosen threshold (such as 0 reads), the attenuation level is recorded and the RF margin can be calculated (i.e. how much power is available in excess of the minimum required to operate the tag) for each 
location tested. Binary response has been used for testing RFID performance within tightly packed items. For instance when testing the readability of tagged objects within a pallet, displaying the read or not response can be used to identify pallet problem areas simply and quickly as done by (Singh, Olsen and Vorst). Another metric for RFID system evaluation is the time it takes for this system to register a read. Studying this measurement can help improve system performance for objects traveling at high speeds it. The most popular method for system evaluation is to record the number of reads registered by the system per unit of time. 


\section{Chapter 3: Methodology}

\section{Task}

In order to determine the RFID doorway portal parameters which maximize system read rate, an experiment was conducted. Various system designs were tested and system performance measured. Based on this work and analysis a proper design of a RFID doorway portal can be chosen to maximize system performance.

\section{Variables}

Various factors with a potential effect on portal performance were considered and six were chosen for this study. Doorway size, antenna placement, reader brand, metallic doorway, tag type, and tag orientation were of interest. The experiment looked at the effect of the six factors on the read rate of a tag as it passed through a doorway portal. Aside from the main effects, interactions were also examined.

\section{Independent (Experimental) Variables}

A summary of the variables studied in this experiment can be found in Table 10 in the Appendix. The independent variables are as follows:

\section{Door Type}

The study looked at the effect of doorway size on the performance of a RFID doorway portal. Specifically if there was going to be a difference in performance between a single and double door sizes. The single door size under study was 36 inches in width and 80 inches in height, and the double door was 72 
inches in width and 80 inches in height. Solidworks models of the PVC frames used in the experiment are presented in Figure 3.

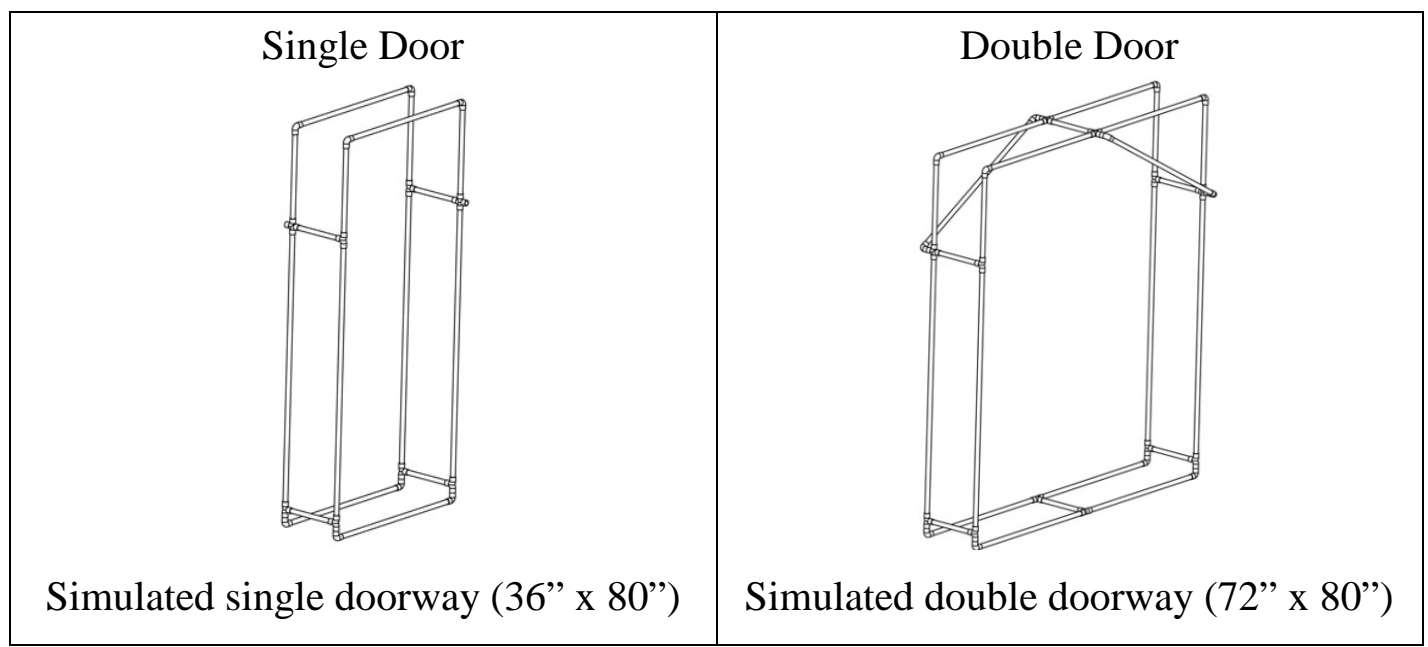

Figure 3: Door sizes (Single/Double)

\section{Antenna Placement}

Five variations of antenna placement were tested. Their orientation and location were determined by the number of antennas present in the doorway. RFID readers have the capability to connect up to four antennas, thus the levels of the factor were variations; testing one, two, three, and four antennas fixed in a doorway. For the single antenna portal setup, the antenna was placed at the top and middle of the doorframe, equal distance from either edge of the frame. For the two-antenna portal, the antennas were placed on opposite sides of the door frame, fixed at half the door height (center of the antenna was $\sim 40$ inches of the ground). A two-antenna variant with one antenna on top and the other on a side was also tested and antennas were positioned in the middle of their respective side. The three-antenna variation of the antenna placement factor was a combination of the one and two-antenna setup. The antennas were placed at the top and sides of the door frame, located in the middle of their respective side. For the final level of the 
antenna placement factor, four antennas were placed on the sides of the door frame, at a half foot distance from the top and bottom of the frame. In order to maximize the potential benefit of this design, the antennas were slightly angled toward the center of the frame at an approximate 27.5 degree angle. The positions for the antennas are displayed below in Figure 4.

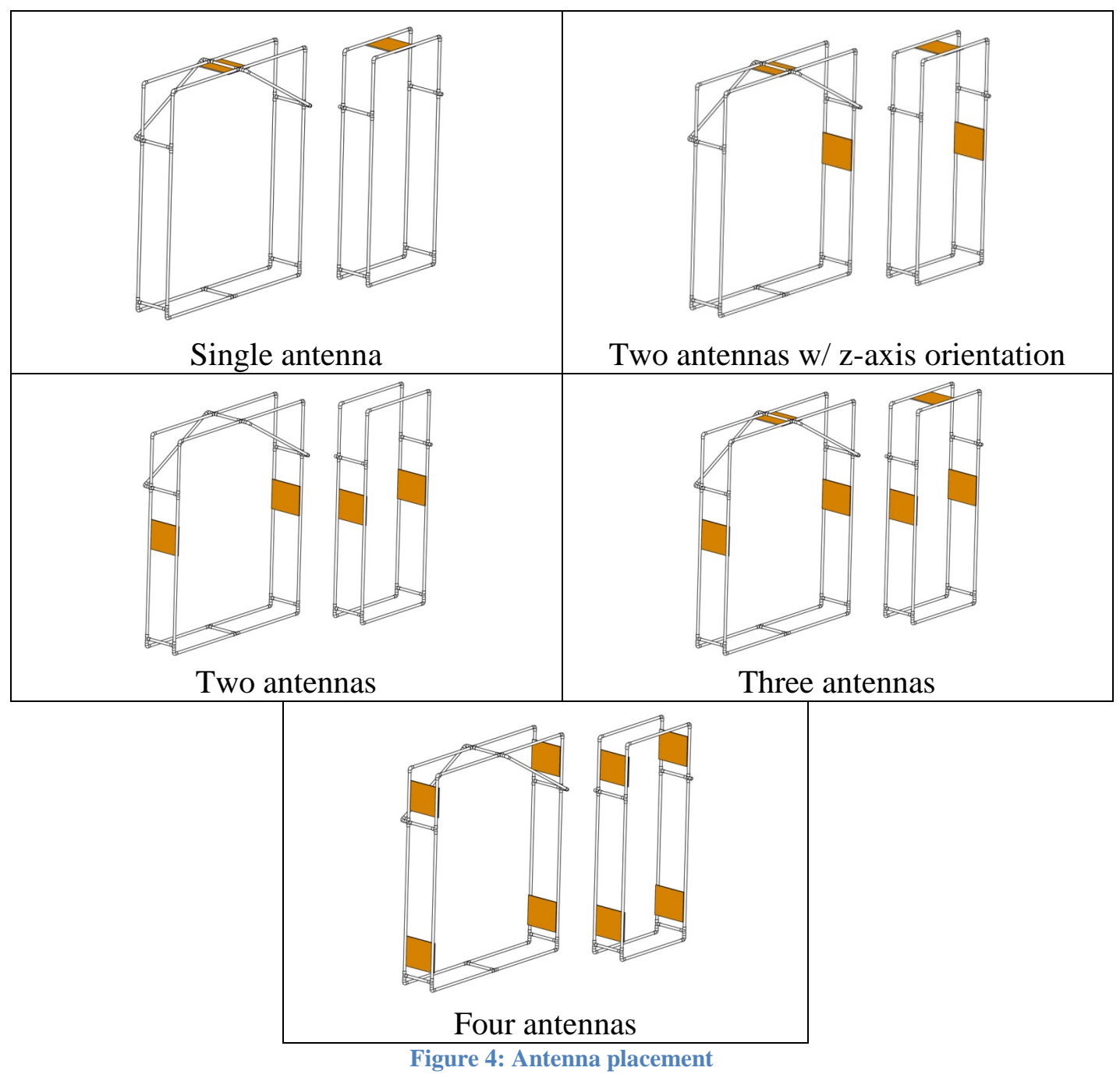

\section{Reader Brand}

Another important aspect of this study was to determine the performance of the two readers, each from a separate manufacturer. The two chosen readers were from Alien and Sirit. 


\section{Metallic Doorway}

The effect of a metal door frame was also simulated and tested.

Experimental runs were conducted with and without an aluminum wire mesh surrounding the frame. To simulate this, the exterior of the PVC frame was covered with the mesh, this resulted in a distance of two inches between the mesh and the back of an antenna. The idea was that the metal mesh would reflect the electromagnetic waves and improve system performance.

\section{Tag Type}

Three tags were incorporated in the experiment to see if one tag performed better than the rest. Two transponders were chosen from Sirit and one from Alien. The main differences of the tags will be discussed further in a later section.

\section{Tag Orientation}

The effect of tag orientation as it passes through the portal was also tested. Tags were put through the doorway in alignment with the $\mathrm{x}, \mathrm{y}$, and $\mathrm{z}$ axis. The $\mathrm{x}-$ axis is horizontal, the $y$-axis is vertical and the $\mathrm{z}$-axis is perpendicular to both the $\mathrm{x}$ and $\mathrm{y}$ axis.

\section{Interactions}

The interaction effects between any two of the six factors were also studied, as it may lead to an improved system design. For instance, the effect of metal surrounding the door frame maybe more prevalent when only one reader antenna is present in the system. 


\section{Dependent Variables}

In order to judge system performance, 27 tags were fixed on three foam boards. On each board a 3 by 3 array of 9 tags was oriented in one of three axes $(\mathrm{x}, \mathrm{y}$, or $\mathrm{z})$. The number of times that each tag was successfully read was recorded as the tags were moved through the portal at a constant speed. A detailed explanation of the experiment setup is provided in a later section.

\section{The Ideal Experiment}

In an ideal situation, the experiment would test the six factors in random order with one tag running through the portal at a time. Given the six factors and the levels to the factors we would be looking at 360 combinations for testing purposes. With 5 replications the total number of runs would be 1800 .

Table 2: Summary of experiment factors

\begin{tabular}{|c|c|c|}
\hline $\begin{array}{c}\text { Independent } \\
\text { Variables }\end{array}$ & Description & Levels \\
\hline Door Type & A double or single doorway & 2 \\
\hline $\begin{array}{c}\text { Antenna } \\
\text { Placement }\end{array}$ & $\begin{array}{l}\text { One antenna at top of door frame, Two antennas on } \\
\text { the sides, Two antennas with one on top of door } \\
\text { frame, antennas both on top and sides, or four } \\
\text { antenna in the corners }\end{array}$ & 5 \\
\hline Reader Brand & Alien or Sirit readers & 2 \\
\hline Metallic doorway & Presence of metal in door frame & 2 \\
\hline Tag Type & Three tag models & 3 \\
\hline \multirow[t]{4}{*}{ Tag Orientation } & Tags positioned in the $\mathrm{x}, \mathrm{y}$, and $\mathrm{z}$ axis & 3 \\
\hline & Runs & 360 \\
\hline & Replications & 5 \\
\hline & Total Runs & 1800 \\
\hline
\end{tabular}


Because there are so many potential factors that affect RFID performance, a large number of experimental cases must be tested in order to evaluate the performance of the RFID components. Ideally, an automated process needs to be used to test these parameters (Zou, Wu and Zhao). Unfortunately, no autonomous process was variable for this study and conducting such a large experiment with randomization of parameters between runs that require setup time is unrealistic. The design of experiment will have to be modified due to labor constraints. The actual experimental design is described in following sections.

\section{Equipment}

In order to conduct the experiment several pieces of equipment were required. Most of the items were fabricated from supplies purchased from a local hardware store. All other equipment was available through the Cal Poly RFID Lab (Poly GAIT). The equipment used were as follows:

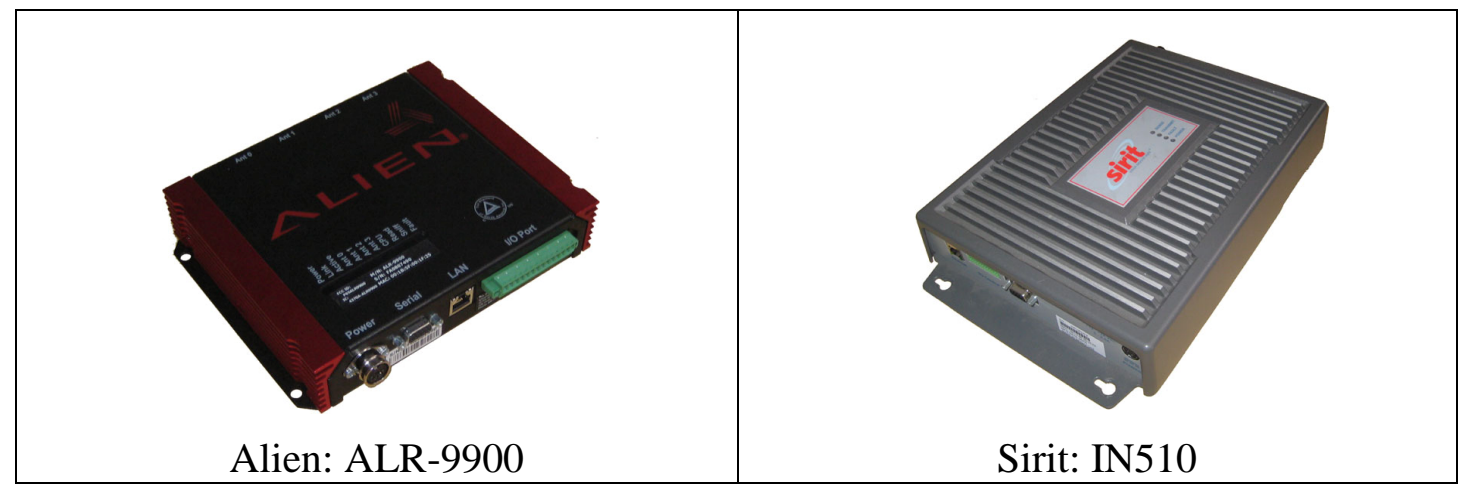

Figure 5: RFID readers 


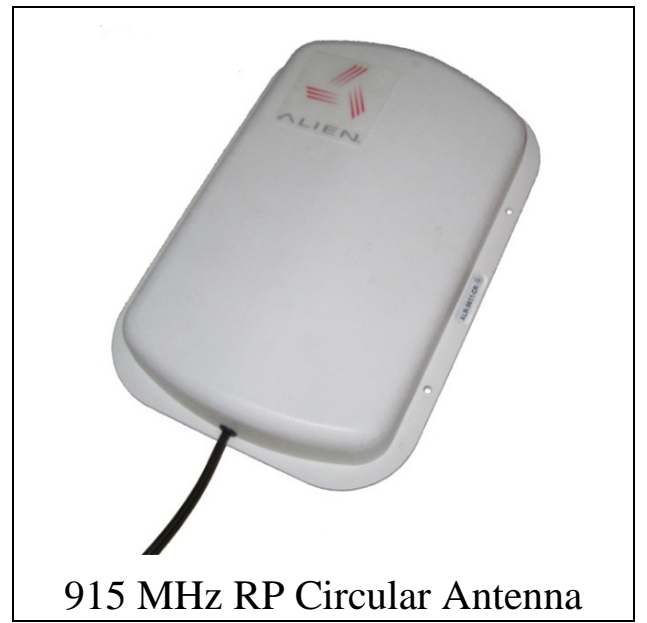

Figure 6: RFID reader antenna

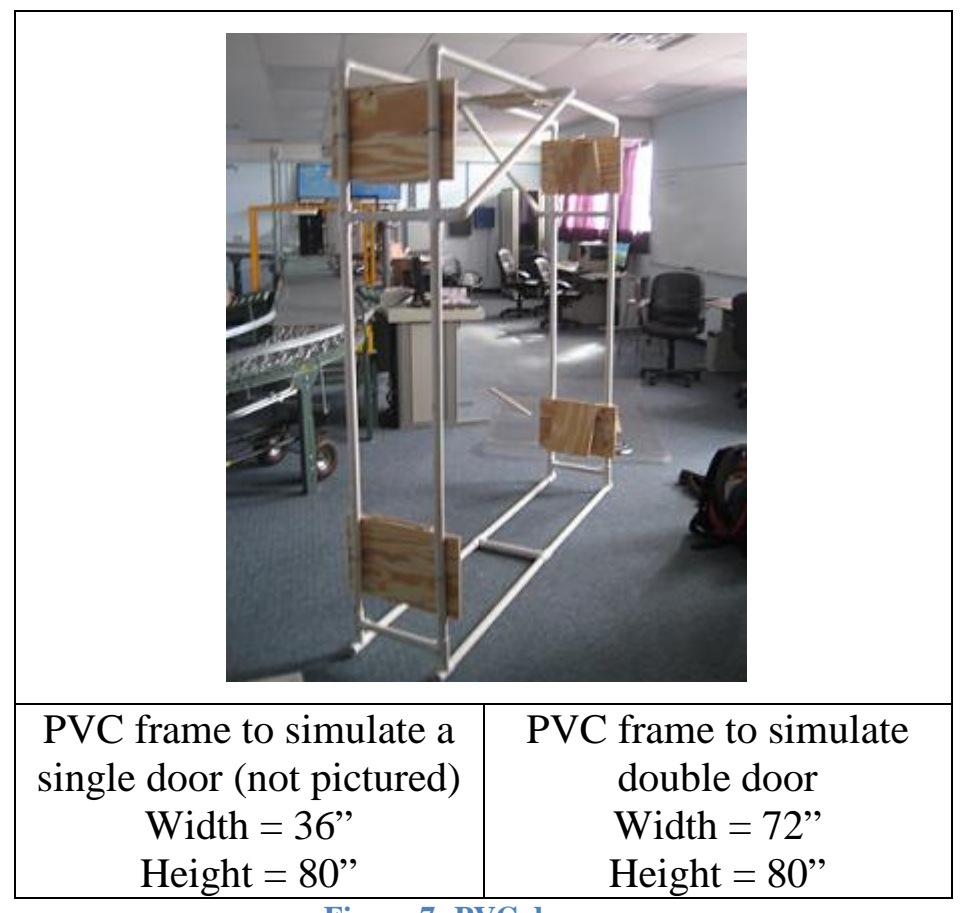

Figure 7: PVC doorway 


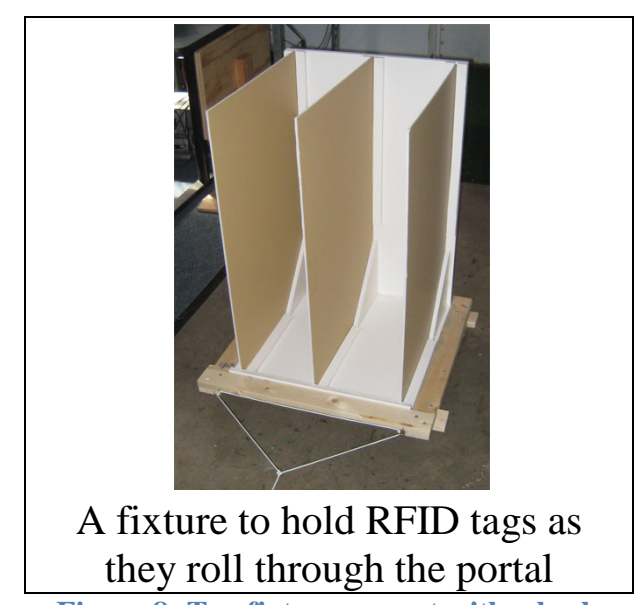

Figure 8: Tag fixture on cart with wheels

The darker color foam boards can be rotated within the white foam board fixture.

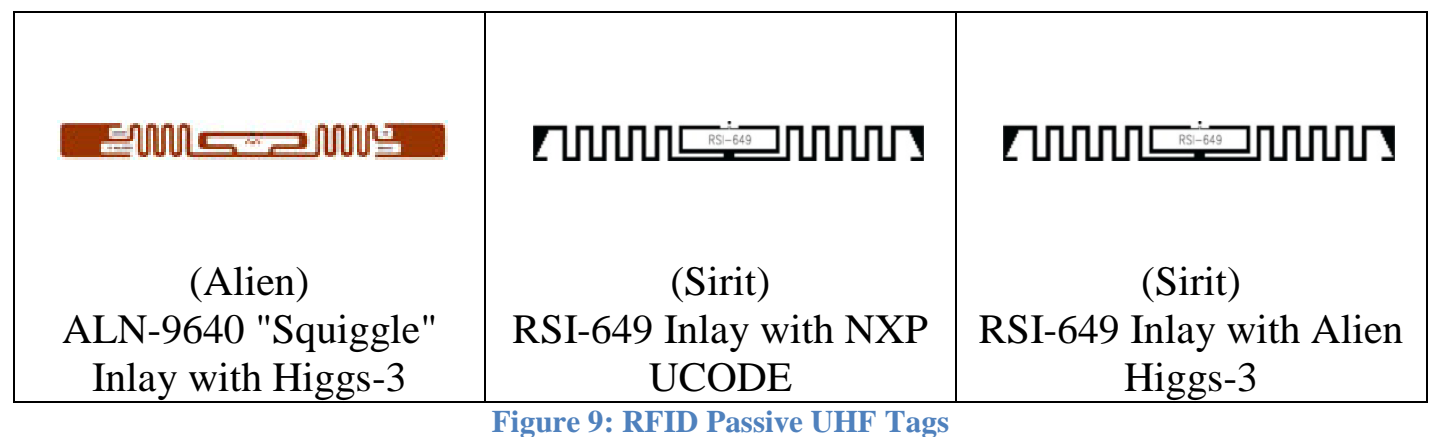

Three passive UHF tags were investigated in the study. All tags were designed for relatively inexpensive multi-purpose use.

- The Alien squiggle inlay with the Alien Higgs-3 IC (chip)

- The Sirit RSI-649 inlay with the NXP UCODE chip

- The Sirit RSI-649 inlay with the Alien Higgs-3 chip

\begin{tabular}{|c|c|c|}
\hline \multicolumn{3}{|c|}{ Other Items } \\
\hline Metal Wire Mesh & $\begin{array}{c}\text { 5 Six Foot tables } \\
\text { (plastic foldable tables } \\
\text { with metal legs) }\end{array}$ & Computer \\
\hline Corded Drill & Ethernet Cable & Extension Cord \\
\hline
\end{tabular}




\section{Design of Experimental Conditions}

With a randomized experiment the run order and replications are all randomly assigned. This means that each experiment observation would have a unique setup. For instance the run order of the experiment may require a double door setup with three antennas, and an Alien reader, on the consecutive run a single doorway with four antennas, with a Sirit reader is required. Under these conditions there is a setup time associated with gathering every observation. The time it takes to rebuild the PVC doorway, orient the antennas, change out the reader and reboot the system at best takes 15 minutes. With 1800 observations 450 hours $\left(\frac{1800 \text { obs } * 15 \text { minutes }}{60 \text { minutes }}\right)$ would be required to conduct the study, and that is still an optimistic number. In order to reduce the amount of time needed to conduct the experiment, two experiment design items were addressed; total amount of runs and the setup time between runs.

\section{Reduce number of runs}

In order to reduce the amount of time required to run the experiment, all the levels of the tag orientation and tag type factors were run at the same time. By doing so a possible bias maybe present in the experiment, where the effect of the tags on each other may sway the results. To combine the two factors, a tag fixture was made out of foam to hold all the tags in the

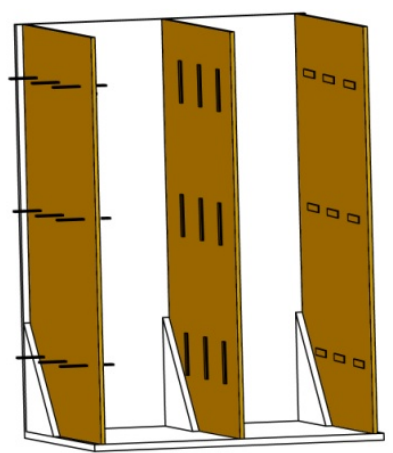

Figure 10: Tag fixture design three orientations. Each of the darker-colored foam boards had tags oriented in 
either $\mathrm{x}, \mathrm{y}$, or $\mathrm{z}$ orientations, with nine tags per board. The tags were positioned at equal spacing to each other, described in Figure 11. The five inch distance between the tags was chosen arbitrarily and the effect of tags on each other at this distance was no considered in this study. There is a good possibility that significant mutual scattering effects will be present at this distance.

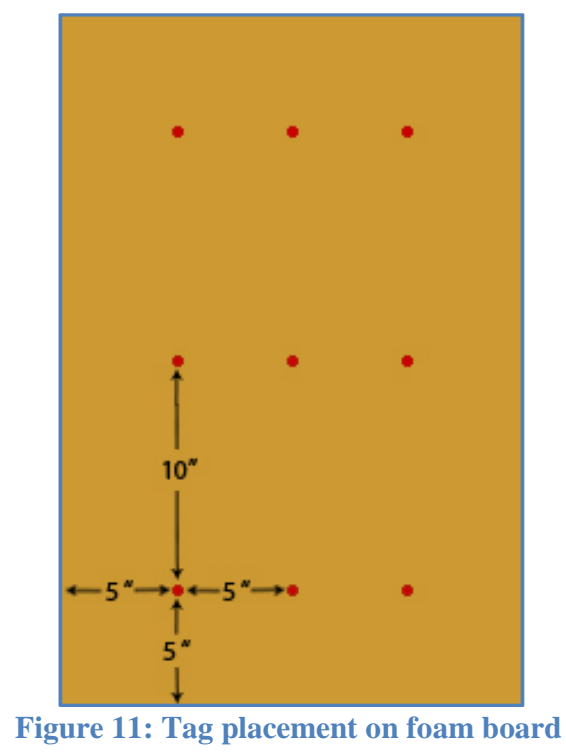

Within the array, nine tags consisting of the three types were randomly placed in the pattern shown in Figure 12 and orientation of the tags was randomly assigned.
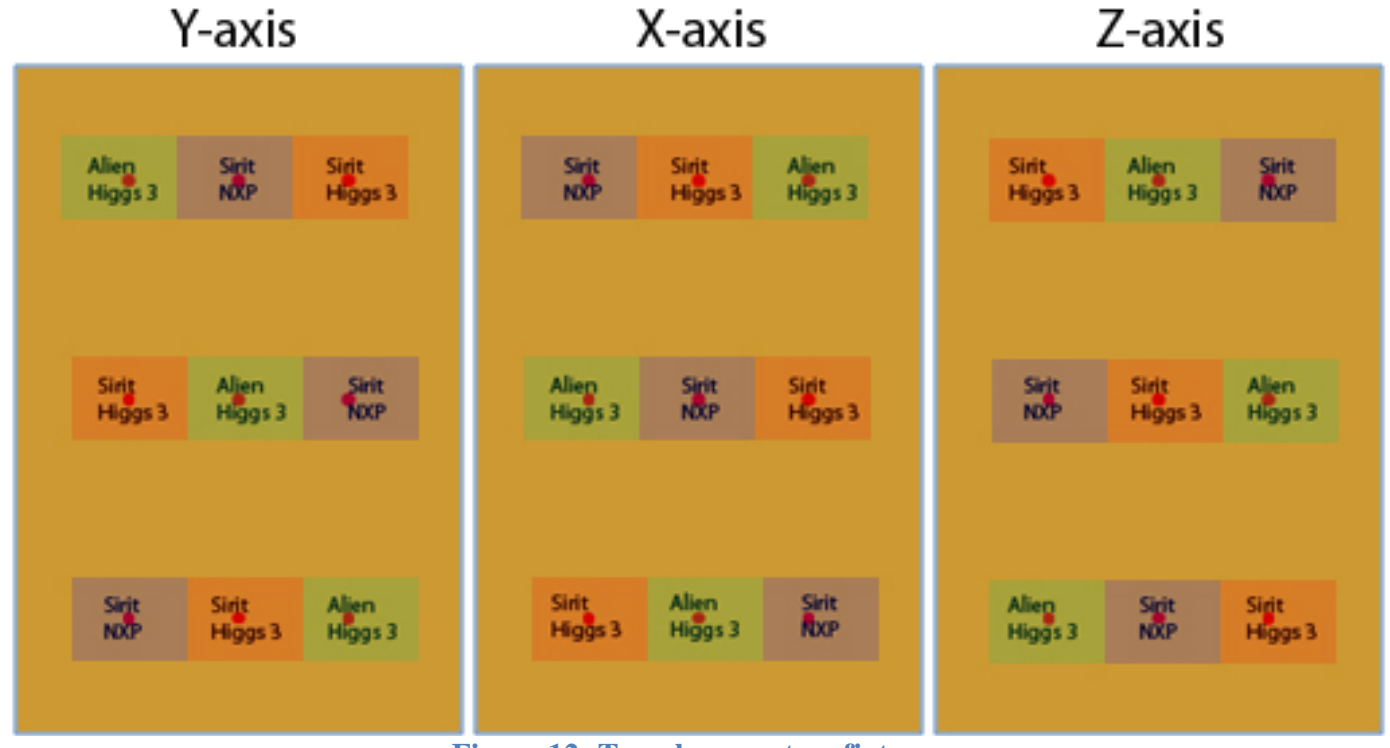

Figure 12: Tag placement on fixture 
With three repeated measures for each tag on every board, along with five replications there are 5400 observations for this experiment

\section{Reduce setup time}

In order to reduce the setup times and decrease the overall time needed to run the experiment, the run order was fixed for three factors. To determine the order of the experimental runs, the independent variables were evaluated in terms of setup difficulty. If the amount of labor and time took longer than three minutes, the setup difficulty of the variable was designated as "Hard". If the setup time was between one and three minutes, the variable was designated as "Medium". If setup time was under a minute, the variable was designated as "Easy".

Table 3: Setup difficulty

\begin{tabular}{|c|c|}
\hline Independent Variables & Setup Difficulty \\
\hline Door Type & Hard \\
Antenna Placement & Medium \\
Reader brand & Medium \\
Metallic doorway & Easy \\
\hline
\end{tabular}

Only the order of the "Easy" factor was randomized throughout the experiment. For the rest of the factors, the levels were randomly chosen and all the replications were then run for that setup. The initial experiment design did not have the ' $2 Z$ ' Antenna Placement level, the scope of the study was broadened to account for the new level, and additional experimental runs were added after the first 160 experimental runs were complete. The order of the "metallic doorway" factor was randomized using the random data feature of Minitab. Please refer to Table 9 in the Appendix for the full experiment run order. 


\section{Reader setup}

Both readers were set to scan for any and all tags in the area at default settings. One of the difficulties of reader comparisons is the difference in firmware settings from one manufacturer to another (Sweeney). To simplify the reader setup, default settings were used with the manufacturer's reader software. Reader power was not attenuated and all the runs were conducted at full power being delivered to the antennas. It is important to note that it has been suggested that evaluation of commercial readers is insufficient due to inconsistencies and high variation of software parameters of the test equipment (Derbek, Steger and Weiss).

\section{Experiment setup}

Four six-foot tables were aligned through the portal; tables were two feet wide with the table top two and a half feet of the ground. The tables acted as a track for the tag fixture cart. The cart was initially positioned $\sim 12$ feet outside the portal, then a drill was used to wind up a string and pull the cart through the portal at a relatively constant rate. The 12 foot distance was chosen because the tags were expected to be outside the read range of the system. Prior to the experiment a test was performed testing the read range of the three tags, in which all three tags were outside the communication zone of the system when the distance between was twelve feet. The twelve foot distance was expected to be outside the range all tags unfortunately the three tag test was not a good representation of a fixture with 27 tags. The diagram of the experiment setup is shown in Figure 13. 


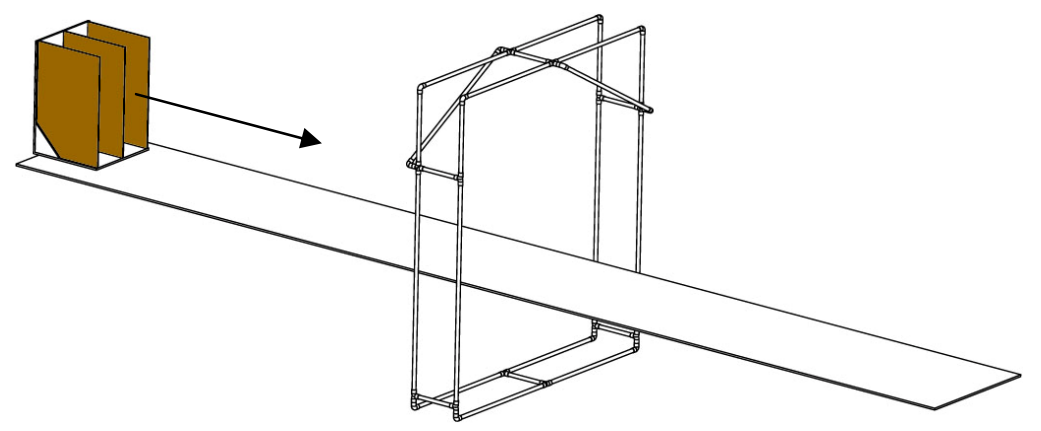

Figure 13: Experiment setup diagram

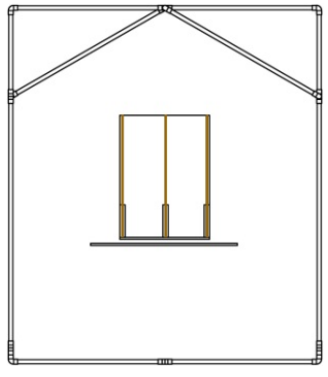

Figure 14: Side view of experiment

The tables were two and a half feet high, and the cart used to roll the tag fixture was and additional two inches. Effectively, the tag fixture was pulled through the middle of the portal, at height of two feet and eight inches. Please refer to Figure 21 in the Appendix for pictures of the actual experiment.

\section{Control Variables}

In order to remove bias from the experiment, various parameters were held constant from one run to another.

\section{Tag speed}

The drill used to pull the fixture at in the experiment had a variable speed, to keep it constant, a clamp was put over the trigger and tightened down until a desired speed was achieved. The average speed of the fixture was calculated to be $1.8 \mathrm{mph}$ or 2.64 feet per second, with a slight variation. A $95 \%$ confidence interval of the speed is between 2.270 and 3.016 feet per second.

\section{Tag location}

Tags were kept at the same distance relative to the frame as they passed through the portal. This distance factor was kept constant from one experimental 
run to the next by aligning the tag fixture with the table, which was aligned with the middle of the portal frame.

\section{Randomized Variables}

In order to reduce bias and avoid confounding variables, certain factors were randomized between experimental runs.

\section{Tag array location}

Since each array board consists of tags oriented in one of the three axes, the foam boards were randomly rotated in the fixture in order to decrease bias. In this case randomization is done to take into account the effect of tag location within the fixture on read rate. For example, if the middle foam board tags performed better than the outer boards, without randomization it would look like one of the tag orientations performed better than the other two.

No software was used to determine the random order of the tag array rotation between runs. Instead after each observation the boards were randomly rearranged at the user's discretion.

\section{Data collection method}

As the tags were pulled through the portal, the reader interrogated the tags. The number of times that a tag was successfully read by the reader was recorded. At the start of each experimental run the tags were positioned twelve feet away from the reader antenna(s), despite this distance some of the tags were still being read before the fixture was set into motion. In order to deal with this obvious experiment design flaw, the response variable data would need to be analyzed and 
redefined before proceeding with the analysis, this way the confounding effects of the reader read range are mitigated.

\section{Hypothesis}

The experiment tested the significance of Doorway Size, Antenna Placement, Reader Brand, Presence of Metal, Tag Type, and Tag Orientation, as well as all the two factor interactions. Several tests of hypothesis were performed as follows:

\section{Main Factors}

For the six main factors let $\beta_{\mathrm{ij}}$ be defined as the effect on the odds of $\mathrm{ij}$ with respect to i1 where,

$\mathrm{i}=$ [Door Type, Antenna Placement, Reader, Metallic, Tag, Tag Orientation] $\mathrm{j}=$ categories in alpha numeric order for the $\mathrm{i}^{\text {th }}$ factor with 1 being the baseline

\section{Hypothesis 1}

Null $\quad \beta_{\text {Doortype }, 2}=0$

Alternative $\quad \beta_{\text {DoorType }, 2} \neq 0$

\begin{tabular}{|c|c|}
\hline \multicolumn{2}{|c|}{ Hypothesis 2} \\
\hline Null & $\beta_{\text {AntennaPlacement }, j}=0$ \\
\hline Alternative & $\beta_{\text {AntennaPlacement }, j} \neq 0$ \\
\hline \multicolumn{2}{|r|}{ Hypothesis 3} \\
\hline Null & $\beta_{\text {Reader,Sirit }}=0$ \\
\hline Alternative & $\beta_{\text {Reader,Sirit }} \neq 0$ \\
\hline \multicolumn{2}{|r|}{ Hypothesis 4} \\
\hline Null & $\beta_{\text {Metallic }, 2}=0$ \\
\hline Alternative & $\beta_{\text {Metallic }, 2} \neq 0$ \\
\hline \multicolumn{2}{|r|}{ Hypothesis 5} \\
\hline Null & $\beta_{T a g, j}=0$ \\
\hline Alternative & $\beta_{\text {Tag }, j} \neq 0$ \\
\hline \multicolumn{2}{|r|}{ Hypothesis 6} \\
\hline Null & $\beta_{\text {Tagorientation }, j}=0$ \\
\hline Alternative & $\beta_{\text {Tagorientation }, j} \neq 0$ \\
\hline
\end{tabular}




\section{Interactions}

With six factors there is a possibility of fifteen unique two way

interactions. Significance of all will be tested and presented in the analysis section. 


\section{Chapter 4: Results}

In this experiment, the read rate or the number of times a tag was successfully read was recorded as a response variable. The collected data set is not included in this report, but is available upon request. For the 5400 observations the lowest registered number of reads was zero and the highest number of reads was 10559, Table 4 provides additional basic descriptive statistics.

Table 4: Basic Descriptive statistics of Read Count

\begin{tabular}{|c|c|c|c|c|c|}
\hline \multicolumn{6}{|c|}{ Descriptive Statistics: Counts } \\
\hline & Total & & & & \\
\hline Variable & Count & Mean & StDev & Minimum & Maximum \\
\hline Counts & 5400 & 84.0 & 452.5 & 0.00 & 10559.0 \\
\hline
\end{tabular}

Figure 15 shows the distribution of the observed read rates for all the tags, please note that the last observation in the histogram is for all reads over 300 .

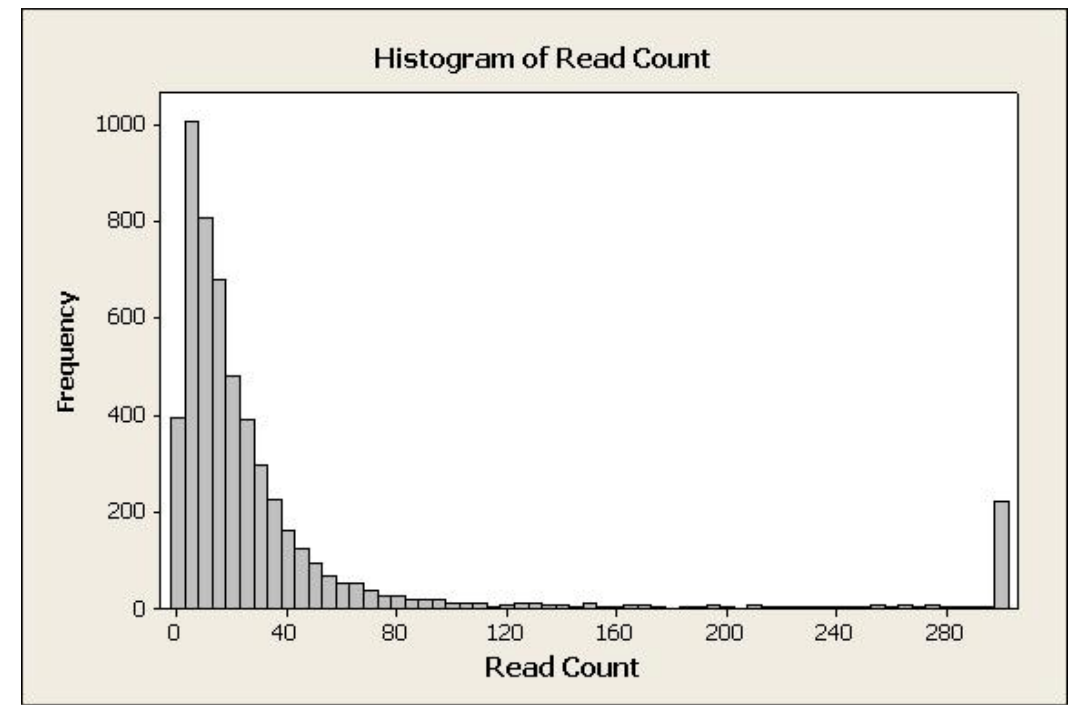

Figure 15: Distribution of read rate

To perform a basic analysis of the high valued read counts, an arbitrary dummy variable was defined a binary with a value of " 1 " for read counts over 300 and " 0 " otherwise. Out the 5400 observations, 221 had read counts in excess of 300 reads. 
The high value "outlying" observations most likely have been the cause of being read prior to the start of each experimental run. A binary regression model was fitted with the newly define dummy variable, and the effects of all factors were tested. Tag Orientation and Tag Type had the highest statistically significant effects on the high valued read counts. Please reference Figure 16 and Figure 17 for more information on the estimated effects of Tag Orientation and Type.

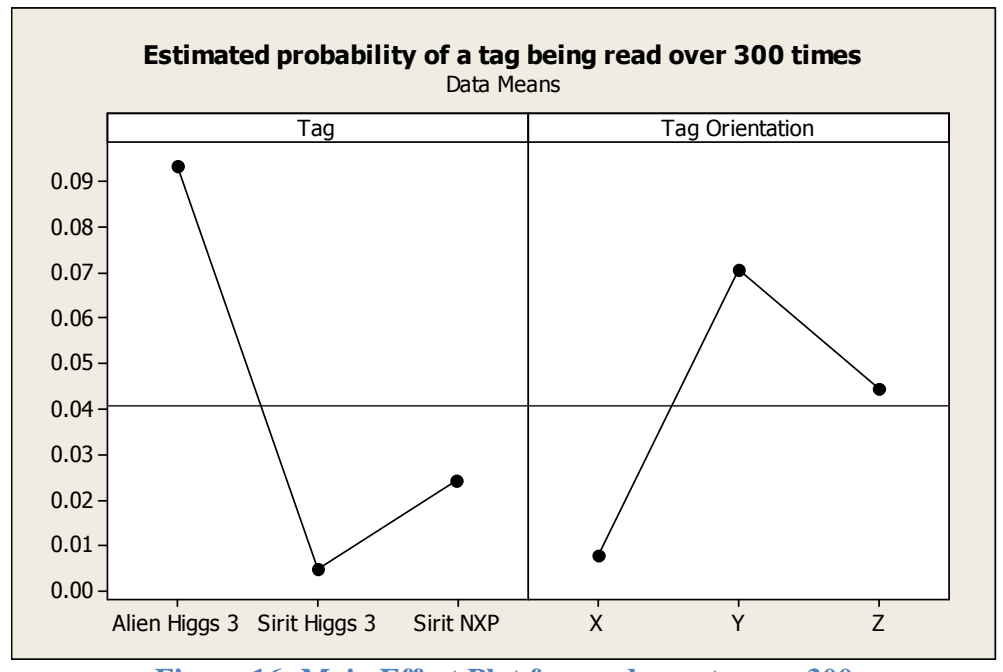

Figure 16: Main Effect Plot for read counts over 300

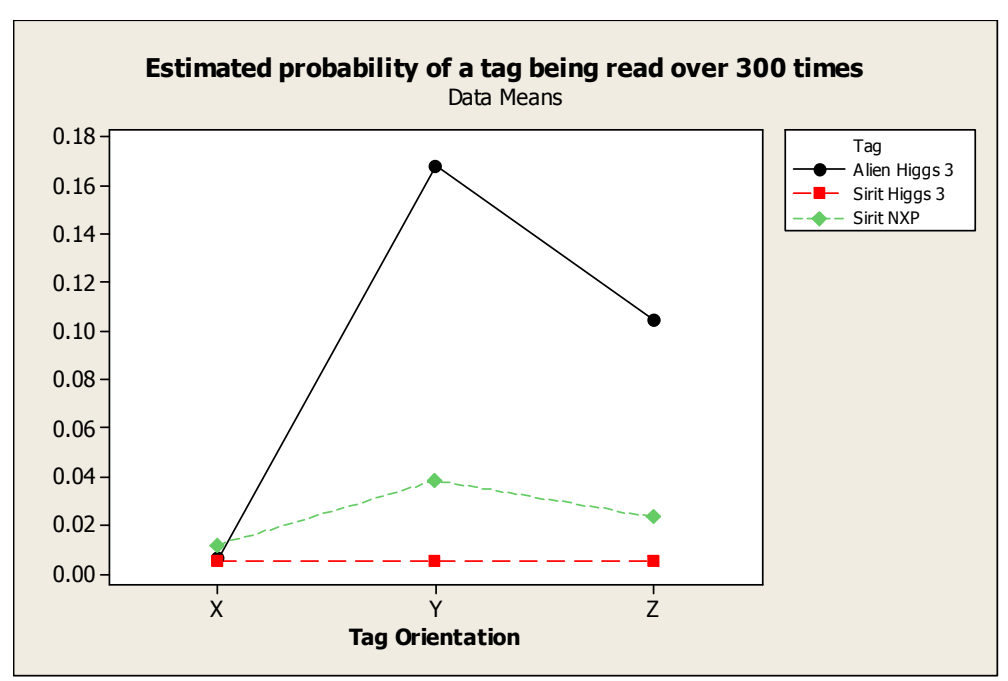

Figure 17: Interaction Plot for read counts over 300 
It appears that the Alien Higgs 3 tags and the tags in the Y-axis orientation were most prone to having reads in excess of 300 . The potential implication of this is the effects of these tags maybe biased in the results of the final analysis.

Along with the high valued outlying observations; the distribution of the data is heavily skewed to the right. This may present a problem when employing statistical tools that depend on normality of the data, in which case the residuals need to be tested to validate the model. 


\section{Chapter 5: Discussion}

\section{Bias}

Throughout the experiment there were several factors that may have been be source of bias. The flaws associated with the experiment are acknowledged as follows:

Software - Performance of each reader may not have been a fair representation of the reader's capability. The difference in software may have caused a significant difference in reader performance as explained in (Sweeney).

The reader software may skew your results because numerous parameters exist within each reader's firmware that are germane to each reader. Tuning these variables to represent identical settings is difficult. This disparity in firmware and firmware settings is one of the difficulties in measuring reader performance on an even playing field.

Wind - The experiment was conducted outside and the effect of wind may have influenced the outcome of the study. The speed of the tag fixture even though controlled by the drill was still variable depending on the wind.

Day - The experiment was carried over a series of five days. Within the day and between days, various weather patterns were observed over the course of the trials (rain, overcast and clear sky) which may have a possible effect on the observed data. Because the experiment was conducted outdoors, tests were run only on days with clear weather and were not run on consecutive days. The varying moister level in the environment may have had an effect on system performance. Tag Fixture Factors - Because the three tag types and three variations of tag 
orientation factors were run through the portal at same time, their effect on each other may be a substantial source of bias.

Fixed Effects - The run order of the explanatory variables was not randomized and can also be a potential source of bias. If the run order was a statistically significant factor then the estimated effects of the predictors would be inaccurate and swayed by the selected run order of the experiment.

\section{Analysis Selection}

The selection of the proper analysis method is dependent on the models ability to deal with outliers and if the data meets all the assumptions of that specific model. Several statistical tools were analyzed for this analysis, such as Poisson Regression, ANOVA, Binary Regression, and Ordinal Logistic Regression.

\section{Poisson Regression}

Under perfect experimental conditions as explained in The Ideal Experiment section of this report, Poisson regression would most likely be the best analytical tool for studying the relationship between read counts and RFID portal system parameters. For this particular experiment, employing Poisson regression will most likely lead to inaccurate estimates of the factor effects. An important requirement of Poisson regression is that the counts occur per constant unit of time. Since, this is not the case for this experiment; the data violates a major assumption of the regression model and hence should not be used as the main analytical tool. 


\section{ANOVA}

Using an ANOVA model to study the effects of the factors on the read rate of the system presents several problems. The presence of the high value observations will have a significant effect on the results and the assumption of normality must be validated.

Despite the presence of the high value "outlying" effects possible ANOVA models were explored. For the first model a General Linear Model was tested for the read count data as a function of the six main effects variables. This model contained 5400 observations, whose residuals were not normally distributed, a clear violation the normality assumption.

Other possible models were explored by averaging the repeated measures, averaging the replication, as well as further aggregation of the data. The idea was that by aggregating the data and taking the averages, the Central Limit Theorem would establish normality. Still, in all cases the residuals of the models were not normally distributed.

For the 1800 observation model the three repeated measures (reads for identical tags on a single tag array) were averaged out. In addition, the five replications were averaged out on top of the repeated measures to give 360 observations. Taking analysis one step further, the Metallic factor was removed and the data was aggregate again. This time there were 180 observations and still the residuals were not normal. Tests of normality are detailed in Figure 18. 

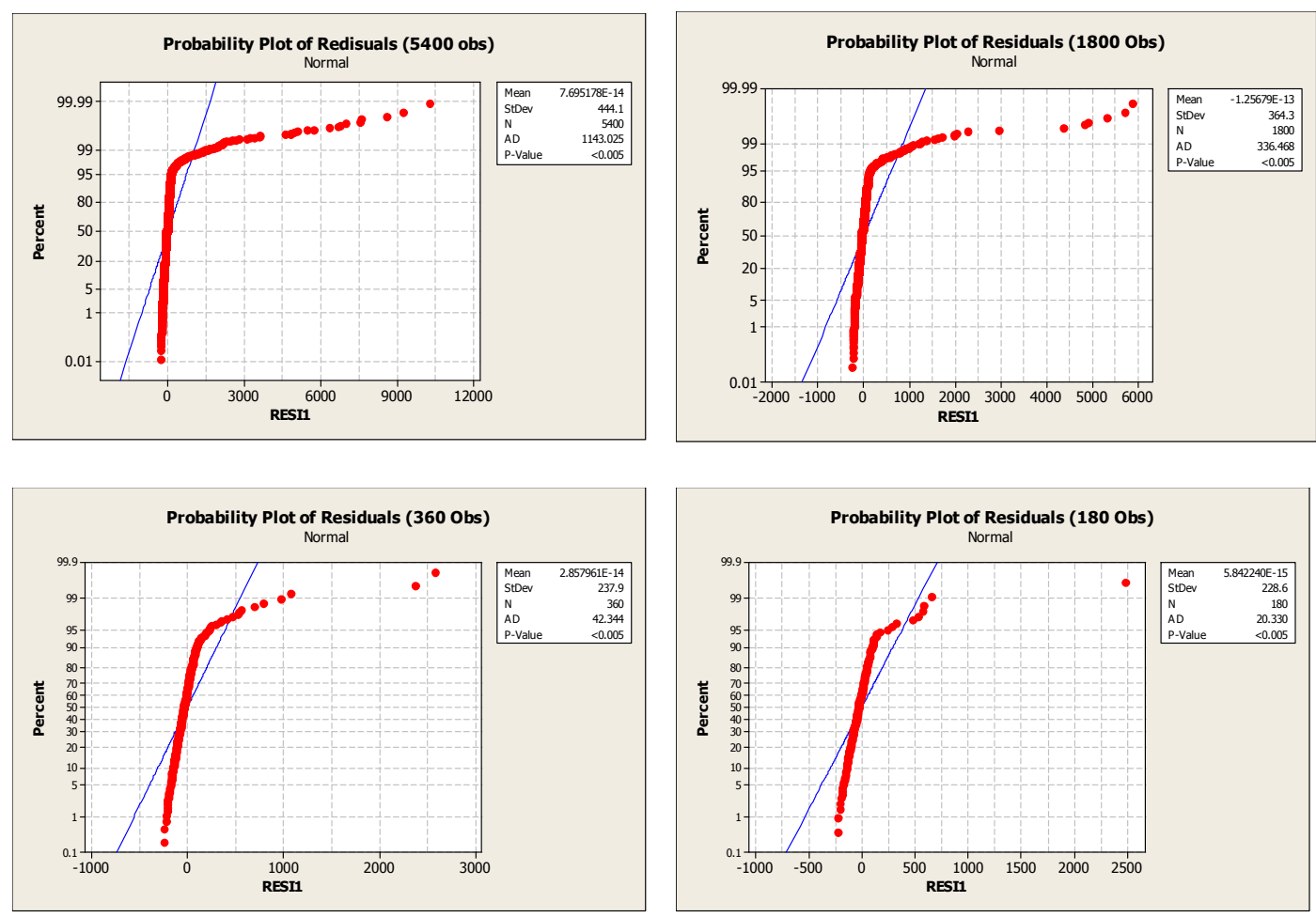

Figure 18: Probability Plots of Residuals

Since, the data does not meet the normality assumption and the model is sensitive to high valued observations, an ANOVA model should not be used for this analysis.

\section{Binary Regression}

The possibility of an ordinary logistic regression model was also explored. By converting the read count data into a binary response of " 1 " for tags that were read at least once and " 0 " otherwise the model is better suited to deal with the high valued outlying observations. For this particular definition of a binary response it does not matter if a tag was read over a thousand times or just once.

Table 5: Binary response variable
\begin{tabular}{|rrr|}
\hline Read & & \\
or Not & Count & Percent \\
0 & 166 & 3.07 \\
1 & 5234 & 96.93 \\
\hline
\end{tabular}


Under the proposed definition of the binary response variable, only three percent of the observations failed to read. With such a low failure rate and high success rate the cell counts for individual levels of the factors would be very sensitive to small changes in the binomial distribution and lead to in accurate estimates. It is harder to estimate effects of predictors well when nearly all the observations are "successes" compared to when there is a similar number of "successes" and "failures" (Agresti).

\section{Ordinal Regression}

An ordinal logistic regression is an extension of binary logistic regression, which may provide a useful model for the analysis of this experiment. By breaking up the continuous response variable into $\mathrm{C}$ categories, and defining the ordinal categories such that each is weighted evenly (each category represents

$\frac{1}{C} * 100$ percent of the observed data) the new response would provide a good representation of the continuous data, but will not be as affected by the high valued observations. This approach should also provide better estimates than a binary model. Hence the analysis of this data will be done with an ordinal logistic regression model. Further explanation of ordinal regression and justification for category selection is provided in the following sections.

\section{Ordinal Logistic Regression}

The most common binary regression is logistic regression or a logit model. Ordinary logistic regression is used to model response variables for which the 
response outcome for each subject is a "success" or a "failure". The logistic regression model has linear form for the logit of this probability,

$$
\operatorname{logit}[\pi(x)]=\log \left[\frac{\pi(x)}{1-\pi(x)}\right]=\alpha+\beta x
$$

Where $\mathrm{X}$ is a single explanatory variable and $\mathrm{Pi}(\mathrm{x})$ denotes the "success" probability of a binary response variable $\mathrm{Y}$ at value of the explanatory variable (Agresti).

When response categories are ordered, logistic regression can utilize the ordering. This case of logistic regression has logits with cumulative probabilities such that:

$$
\begin{gathered}
\operatorname{logit}[\operatorname{Pr}(Y \leq j)]=\log \left[\frac{\operatorname{Pr}(Y \leq j)}{1-\operatorname{Pr}(Y \leq j)}\right] \\
j=1, \ldots, J-1
\end{gathered}
$$

Where $\mathrm{Y}$ is the response categories with counts that have a multinomial distribution and $\mathbf{J}$ is the last ordered category.

By defining the cut off for the categories in Y such that the distribution is fairly evenly spread among four or more categories the efficiency loss (in the sense that larger standard error result) will be minor and offer a better model as compared to a binary regression (Agresti).

Because of the strong connection between the $\mathrm{J}$ defined categories and the continuous distribution of the read counts, the categorical variable is expected to satisfy a model with the same linear predictor. This implies that a proportional odds model should be assumed. Meaning that it requires only a single parameter rather than $\mathrm{J}-1$ parameters to describe the effect of a factor or in other words the 
effect of a parameter should be invariant to choice of categories for Y. This Also implies that if two researchers who use different response categories in studying a predictor's effect should each reach similar conclusions (Agresti). Hence, selection of the number of categories would not have an effect on the results.

\section{Model Selection}

A new ordinal category response variable was defined from the recorded read count data. The new response was used to build an Ordinal Logistic regression model as a function of the system design parameters. To develop the model a process of backwards elimination was used.

\section{Definition of the Ordinal Response}

The continuous data of the read counts was divided into five categories, such that all the data was spread evenly across. This was accomplished by finding the 20th, 40th, 60th and 80th percentiles. The first category of the ordinal response was set as less than or equal to the 20th percentile. The second category was set as above the 20th and up to the 40th percentile. The third was set as above the 40th and up to the 60th percentile. The fourth category was defined as above the 60th and up to the 80th percentile, and the fifth group was defined as above the 80th percentile. The resulting categories of the new ordinal response were defined as presented in Table 6.

Table 6: Definition of the Ordinal Response
\begin{tabular}{|c|c|}
\hline Category & Cutoff Definition \\
\hline A & 0 reads to 6 reads \\
\hline B & 7 reads to 12 reads \\
\hline C & 13 reads to 21 reads \\
\hline D & 22 reads to 39 reads \\
\hline E & 40 reads or higher \\
\hline
\end{tabular}


Under these definitions the spread of data among the five categories was fairly even, with each category representing $\sim 20 \%$ of the data. For more information please reference Table 7.

Table 7: Tally of Ordinal Categories
\begin{tabular}{|rrr|}
\hline ORDINAL & Count & Percent \\
A & 1205 & 22.31 \\
B & 999 & 18.50 \\
C & 1058 & 19.59 \\
D & 1083 & 20.06 \\
E & 1055 & 19.54 \\
N= & 5400 & \\
\hline
\end{tabular}

\section{Backwards Elimination}

The process of backwards elimination was used to develop the ordinal logistic regression model. Model selection began with a complex model of the six main variables and their fifteen two way interaction terms. The model was run, the highest p-value (insignificant) interaction term was removed, and the process was repeated until all remaining interaction terms were of statistical significance. All the main factors were kept in the model regardless of their significance, in order to reduce bias in estimating the effects of other predictors (Agresti).

\section{Significance of the Day (Run Order)}

Since the run order of the experiment was fixed and the experiment conducted over a series of five days, the results may have been swayed by the effect of a 'Day' variable. Meaning that one day for reasons unknown resulted in better than average performance, which may have led to increased performance observations of the parameters tested that day. To test the significance of whether or not day had an effect on the observed results, an ordinal logistic regression model was studied with a single predictor of 'Day'. Running an ordinal logistic 
regression with 'Day' as the predictor shows that there was a statistically significant effect ( $\mathrm{p}$-value $<0.0001$ ). With observations from day one having the highest observed performance. Please note that only the Alien reader with a single antenna was the only configuration tested on day one, and may lead to inaccurate and inflated performance estimates for an Alien system with one antenna.

The run order of the 200 experimental runs was also tested, and proved to be statistically significant with a P-value $<0.0001$. The implication of this is that the lack of a randomized run order presents a flaw with the experiment design that will lead to inaccurate estimates of the effects. Despite obvious flaws with the data, statistical analysis was performed.

\section{Model}

The variation of the response variables was studied as a function of Door Type, Antenna Placement, Reader, Metallic, Tag, and Tag Orientation factors.

\section{Main Factors}

As a first step in the analysis of the data, a model consisting of only the main factors was studied. All factors except "Metallic" were of statistical significance, please reference Table 11 for a detailed Minitab output. To quantify the relationships logistic regression results are communicated though odds and odds ratios. Where an odds is the probability of the event occurring divided by the probability that the event doesn't occur. An odds ratio is calculated by dividing the odds of the event by the odds of a baseline. Hence, the fraction will provide the percent increase in the odds of the event relative to the baseline. 
Looking at the relationship between the response and the Door Type factor shows that the odds of a double doorway are less than that of a single doorway with an odds ratio of .77 when a single doorway is the baseline $\left(\frac{\text { odds(Double })}{\text { odds(Single })}=\right.$ .77). The odds ratio can be rewritten with respect to the double doorway by taking the inverse of the odds ratio $\left(\frac{\text { odds }(\text { Single })}{\text { odds(Double })}=\frac{1}{\frac{\text { odds(Double) }}{\text { odds(Single })}}=\frac{1}{.77}=1.2987\right)$ meaning that the odds of being at the low end of the read count scale is $29 \%$ higher for a single doorway than a double doorway. An interpretation of these results indicates that a double door is expected to have higher read counts and hence perform better.

In order to simplify the interpretation process of the model parameters, SAS was used to conduct the analysis with the ordinal response category order reversed. Now instead of interpreting the odds of an event being at the lower end of the read count scale in respect to the baseline, interpretation can be done on the odds of an event being at the higher end of the scale. A summary of the estimated parameters is presented below. Please refer to Table 12 for the SAS output.

- The odds of a double doorway being at the higher end of the read count scale are $29.1 \%$ higher than that of a single doorway.

- A single antenna portal performed significantly better than the ' 2 ' and ' 4 ' antenna placement categories. While there was no significant difference between a single antenna and the ' $2 Z$ ' and ' 3 ' antenna placement categories.

- The odds of a Sirit reader being at the higher end of the read count scale are $37.8 \%$ than that of an Alien reader. 
- The presence of metal in the doorway as tested was not a significant factor.

- The Sirit NXP tag performed the best, followed by the Alien Higgs 3 tag, and the Sirit Higgs 3 tag performed the worst. The low performance of the Sirit Higgs 3 inlay is most likely attributed to the inlay material. The sample of the Sirit Higgs 3 tags used in this experiment had an aluminum inlay, while future productions of the tag would most likely be with silver (Canaris).

- Tags oriented in the Y axis performed the best, while there was no significant difference between the $\mathrm{X}$ and $\mathrm{Z}$ axis oriented tags.

\section{Interactions}

By incorporating interaction effects into the model, a better understanding of the effects can be established. Out of the fifteen possible interaction terms, eight were of statistical significance at $\alpha=0.01$. Please refer to Table 13 in the appendix for the SAS output of the eight interaction term model. The significant interactions are as follows:

\begin{tabular}{|l|l|}
\hline 1 & Door_Type*Antenna_Placement \\
\hline 2 & Door_Type*Reader \\
\hline 3 & Door_Type*Tag_Orientation \\
\hline 4 & Antenna_Placement*Reader \\
\hline 5 & Antenna_Placement*Tag \\
\hline 6 & Antenna_Placement*Tag_Orientation \\
\hline 7 & Reader*Tag_Orientation \\
\hline 8 & Tag*Tag_Orientation \\
\hline
\end{tabular}

To develop a simpler model for interpretation only the terms with an extremely strong level of significance were kept in the model. Based on the Wald ChiSquare statistics three terms with the highest significance level were selected. For this approach a higher Wald statistic represents a lower P-value. The Antenna 
Placement*Tag Orientation interaction term has a substantially higher test statistic, and appears to be the most significant interaction term for the model. For more information please refer to Table 8 .

Table 8: SAS Type 3 Analyses of Effects

\begin{tabular}{|lrrr|}
\hline Effect & DF & \multicolumn{1}{l|}{ Wald } \\
Chi-Square & Pr $>$ ChiSq \\
Door_Type*Antenna_Pl & 4 & 82.7157 & $<.0001$ \\
Door_Type*Reader & 1 & 74.6071 & $<.0001$ \\
Door_Type*Tag_Orient & 2 & 11.6483 & 0.0030 \\
Antenna_Place*Reader & 4 & 161.6622 & $<.0001$ \\
Antenna_Placemen*Tag & 8 & 53.2067 & $<.0001$ \\
Antenna_P*Tag_Orient & 8 & $\mathbf{1 0 8 0 . 5 6 1 5}$ & $<.0001$ \\
Reader*Tag_Orientati & 2 & 45.2464 & $<.0001$ \\
Tag*Tag_Orientation & 4 & $\mathbf{2 2 9 . 2 8 8 6}$ & $<.0001$ \\
\hline
\end{tabular}

\section{Final Model}

The final ordinal regression model includes the six main factors and three interaction terms. The selected interactions were Antenna Placement*Reader, Antenna Placement*Tag Orientation, and Tag*Tag Orientation. Please reference Table 14 in the Appendix for the SAS output of the final model.

The interpretation of interactions presents a challenge, especially in the case of ordinal logistic regression. Due to the nature of the logit models, interpretations are done by looking at the effect on the odds of an event occurring with respect to the odds of a baseline.

For this model, the baseline group is set to an Alien system, setup in a single doorway with one antenna, using an Alien Higgs 3 tag oriented in the $\mathrm{X}$ axis. All other system design configurations are compared in their effect on the odds relative to the baseline configuration. For a model with no interaction terms the effect on the odds of one factor is assumed to be constant for all levels of the other factors present in the model. When multiple interaction terms are present 
such as in this case, it becomes a lot more difficult to interpret the meaning of the interactions.

To simplify the interpretation and summarize the results of the analysis, SAS was used to generate estimated probabilities of the selected final model. With the assumption of proportional odds, in which the effects on the odds of the predictors are constant between all levels of the ordinal category, the fitted probabilities were plotted against the model factors, to display a relative performance comparison of the interaction terms. An interaction plot was created and is displayed in Figure 19. Please note that the grey interactions were not part of the model.

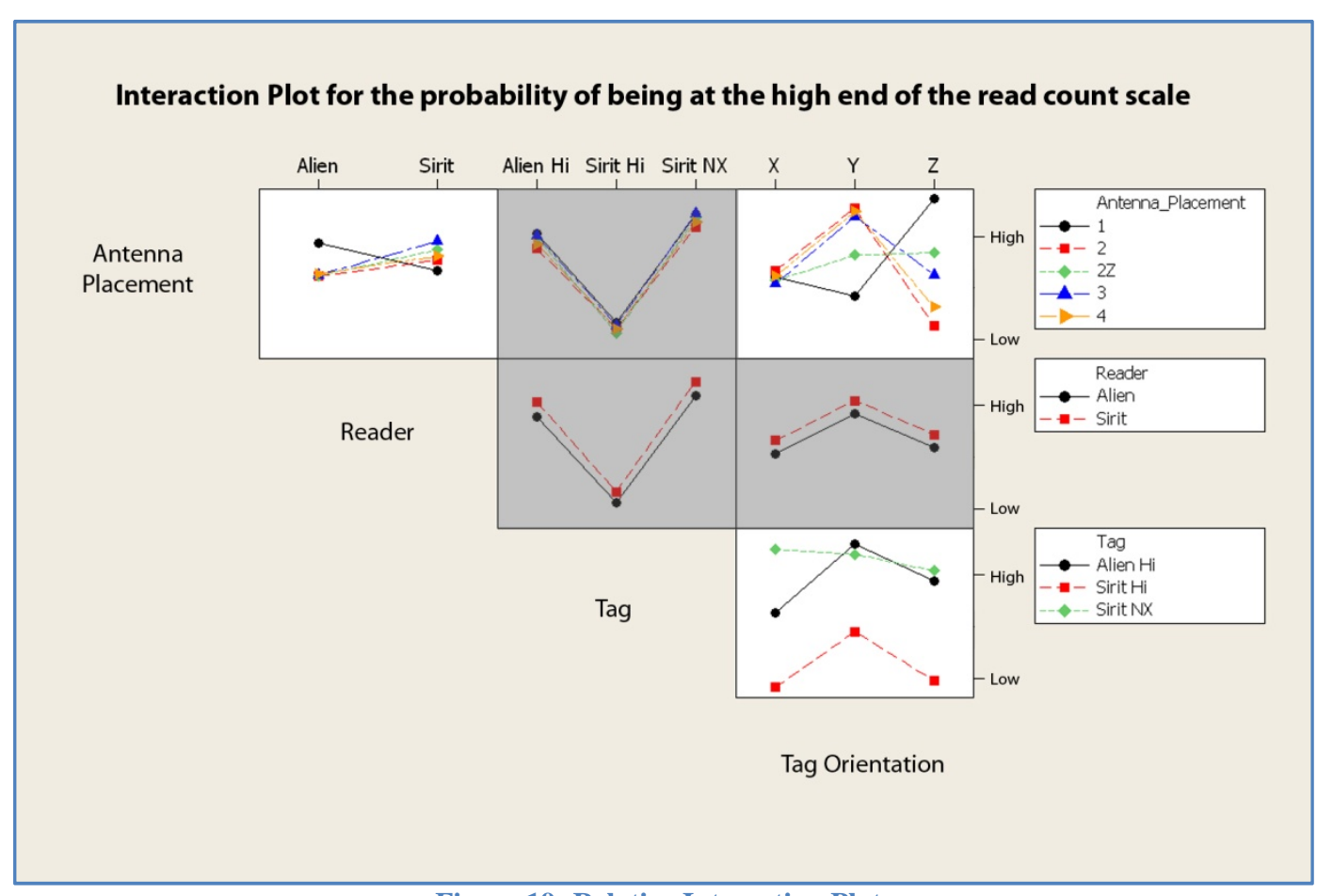

Figure 19: Relative Interaction Plot

- For the Antenna Placement*Reader interaction, with the exception of a single antenna, a Sirit system is expected to have a higher probability of being in the higher end of the read count scale than that of an Alien 
system. The observed performance of a single antenna may be due to the Day factor as described in an earlier section.

- For the Antenna Placement*Tag Orientation interaction, there was a huge difference in performance between the three tag orientation axes. Most notably performance of antenna configurations had the highest variation in the Z-axis, with the ' 1 ', '2Z', and ' 3 ' Antenna Placement levels performing the best. This was expected because only the ' 1 ', ' $2 Z$ ', and ' 3 ' antenna placement levels had a top mounted antenna that was parallel to the Z-axis tags. The particular order of expected performances of the antenna placement parameters with $\mathrm{Z}$ axis oriented tags indicates that a top mounted antenna will provide a higher read rate for the Z-axis. Also, the results of this analysis implies that with every additional antenna in the system that is not oriented parallel to the Z-axis, performance will decrease for the $\mathrm{Z}$ axis oriented tags. This was also expected because, when multiple antennas are connected to a reader, the reader software will cycle through the antennas one by one. Decreasing the amount of time that any single antenna has to communicate with a tag. As expected the single antenna performed the best in the Z-axis followed by the ' $2 \mathrm{Z}$ ' and the ' 3 ' antenna configurations.

- Another important aspect of the Antenna Placement*Tag Orientation interaction is the performance differences between the $\mathrm{X}$ and $\mathrm{Y}$ axis oriented tags. Since the experiment used circularly polarized antennas one would expect that the $\mathrm{X}$ and $\mathrm{Y}$ oriented tags would have similar performance. Instead there is a huge difference with the $\mathrm{Y}$-axis tags

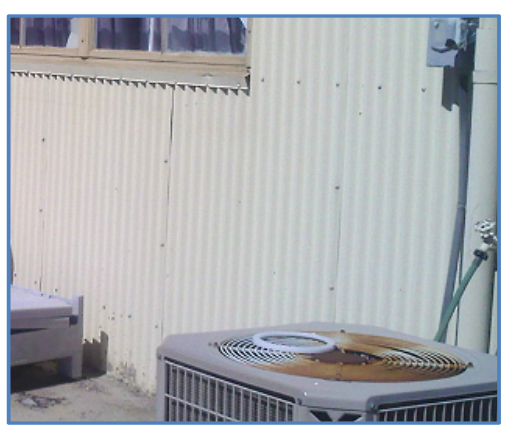

Figure 20: Building side exhibiting far higher variation in performance. This effect is most likely caused by the environment. On one side of the experiment location the presence of a wavy metal building 
exterior may have been responsible for the performance difference between the $\mathrm{X}$ and $\mathrm{Y}$ axes.

- For the Tag*Tag Orientation interaction, performance of the NXP tag was better in the $\mathrm{X}$-axis than any other tag. Interpretation of this interaction seems to indicate that the NXP has the lowest amount of variation among the test orientations and on average is expected to perform the best. 


\section{Chapter 6: Conclusion}

In conclusion the results of the experiment and the presented analysis method can be used for the selection of passive UHF RFID doorway portal systems in which orientation of tag objects is not controlled. Examples of such RFID implementations are equipment or tool rooms with other applications where movement of inventory items should be reported. Since the orientation of tags going through such a RFID system are not controlled, system design parameter selection becomes important for achieving a high read rate for tags in all possible orientation.

The results of this study indicate that the expected probabilities of a system being in the higher end of the read count scale are maximized under the following parameters.

- A double doorway system will perform better than a single doorway portal

- The '1', '2Z', and ' 3 ' levels of the Antenna Placement factor performed the best with no significant difference between the three.

- A Sirit reader performed better than an Alien system, with both readers tested at default settings.

- The presence of metal as tested was found to have no statistically significant effect on the readability of tags in the system.

- The Sirit NXP tag performed the best, with the Alien Higgs 3 tag showing very similar performance characteristics.

- For an RFID system to have good performance for tags in a variety of orientations, antennas should be placed at the top of the doorway as well as the sides. 
Based on this analysis, a Sirit system, setup in a double doorway, with an antenna at the top of the frame as well as the sides, using Sirit NXP tags is expected to have the highest performance level out of the parameters studied. 


\section{Chapter 7: Recommendations}

This study was conducted with the intention of using the developed information to integrate RFID technology in a tool room environment. Even though the experiment has provided significant insight into developing such a portal, the study simplified various factors which may have had a significant effect on system performance and should be studied further.

\section{Future Studies}

\section{Automation}

Future studies should be conducted using an automated procedure to gather data. This way a large sample size of data can be collected. Also, by using an automated process the duration of tag exposure inside the portal can be finely controlled and recorded at a high speeds. One of the biggest issues with this experiment was the lack of randomization of the tested factors. By employing an automated process to gather the data, the experiment can be designed to test all variables of interest with a fully randomized design. Such information may provide the basis for a Poisson Regression Model. This analysis approach should provide better estimates for the effects than an ordinal logistic regression model.

\section{Attenuation}

This study did not look at the potential effect of attenuation on system performance. When developing a tool room portal the engineer will likely be required to attenuate the power to the reader antennas. If the reader antennas were to be used at full power and the tools in storage were relatively close to the portal, the system may pick up tags before they have been passed through the portal, a 
problem encountered in this experiment. Reader antennas operating at an attenuated power level may affect the read rate and other predictors of the system.

\section{Actual Tools}

As described in the background section of the report, the factor that makes RFID implementation difficult is the effect of the environment on the system. Since the main items that would be tagged in a tool room are going to be primarily made of metal they will most likely have a direct effect on the performance of the system. Further study or a pilot program should be conducted, testing system performance with tags on metal tools

\section{Temperature and Humidity}

Temperature and humidity may be significant factors and their effects should be explored in future studies. As mentioned earlier in the report, moisture has a substantial effect on RFID systems. Performance of an external doorway portal as compared to an interior doorway maybe completely different given the environment and should be explored further. 


\section{Appendix}

\section{Definitions}

RFID -Radio Frequency Identification

Pilot - A early development phase of RFID implementation, used to evaluate performance before committing to heavy investments

ITV - In-transit visibility

Transponder - A device that transmits a predetermined message in response to a specific signal (tags)

Reader - A device that emits radio waves to RFID tags within range

EPC - Electronic Product Code

UPC - Universal Product Code

DoD - Department of Defense

CIO - Chief Information Officer

FCC - Federal Communications Commission

IC - Integrated Circuit 


\section{Tables and Figures}

Table 9: Experimental Runs

\begin{tabular}{|c|c|c|c|c|}
\hline Run & $\begin{array}{l}\text { Door } \\
\text { Type }\end{array}$ & $\begin{array}{c}\text { Antenna } \\
\text { Placement }\end{array}$ & Reader & Metallic \\
\hline 1 & 2 & 1 & Alien & Yes \\
\hline 2 & 2 & 1 & Alien & Yes \\
\hline 3 & 2 & 1 & Alien & No \\
\hline 4 & 2 & 1 & Alien & No \\
\hline 5 & 2 & 1 & Alien & No \\
\hline 6 & 2 & 1 & Alien & Yes \\
\hline 7 & 2 & 1 & Alien & No \\
\hline 8 & 2 & 1 & Alien & Yes \\
\hline 9 & 2 & 1 & Alien & No \\
\hline 10 & 2 & 1 & Alien & Yes \\
\hline 11 & 1 & 4 & Alien & No \\
\hline 12 & 1 & 4 & Alien & Yes \\
\hline 13 & 1 & 4 & Alien & Yes \\
\hline 14 & 1 & 4 & Alien & Yes \\
\hline 15 & 1 & 4 & Alien & No \\
\hline 16 & 1 & 4 & Alien & No \\
\hline 17 & 1 & 4 & Alien & No \\
\hline 18 & 1 & 4 & Alien & No \\
\hline 19 & 1 & 4 & Alien & Yes \\
\hline 20 & 1 & 4 & Alien & Yes \\
\hline 21 & 2 & 2 & Sirit & Yes \\
\hline 22 & 2 & 2 & Sirit & No \\
\hline 23 & 2 & 2 & Sirit & No \\
\hline 24 & 2 & 2 & Sirit & Yes \\
\hline 25 & 2 & 2 & Sirit & Yes \\
\hline 26 & 2 & 2 & Sirit & No \\
\hline 27 & 2 & 2 & Sirit & Yes \\
\hline 28 & 2 & 2 & Sirit & Yes \\
\hline 29 & 2 & 2 & Sirit & No \\
\hline 30 & 2 & 2 & Sirit & No \\
\hline 31 & 2 & 4 & Sirit & Yes \\
\hline 32 & 2 & 4 & Sirit & No \\
\hline 33 & 2 & 4 & Sirit & No \\
\hline 34 & 2 & 4 & Sirit & Yes \\
\hline 35 & 2 & 4 & Sirit & Yes \\
\hline 36 & 2 & 4 & Sirit & No \\
\hline 37 & 2 & 4 & Sirit & Yes \\
\hline
\end{tabular}




\begin{tabular}{|c|c|c|c|c|}
\hline 38 & 2 & 4 & Sirit & Yes \\
\hline 39 & 2 & 4 & Sirit & No \\
\hline 40 & 2 & 4 & Sirit & No \\
\hline 41 & 1 & 3 & Alien & Yes \\
\hline 42 & 1 & 3 & Alien & No \\
\hline 43 & 1 & 3 & Alien & No \\
\hline 44 & 1 & 3 & Alien & Yes \\
\hline 45 & 1 & 3 & Alien & Yes \\
\hline 46 & 1 & 3 & Alien & No \\
\hline 47 & 1 & 3 & Alien & Yes \\
\hline 48 & 1 & 3 & Alien & Yes \\
\hline 49 & 1 & 3 & Alien & No \\
\hline 50 & 1 & 3 & Alien & No \\
\hline 51 & 2 & 3 & Sirit & Yes \\
\hline 52 & 2 & 3 & Sirit & No \\
\hline 53 & 2 & 3 & Sirit & No \\
\hline 54 & 2 & 3 & Sirit & Yes \\
\hline 55 & 2 & 3 & Sirit & Yes \\
\hline 56 & 2 & 3 & Sirit & No \\
\hline 57 & 2 & 3 & Sirit & Yes \\
\hline 58 & 2 & 3 & Sirit & Yes \\
\hline 59 & 2 & 3 & Sirit & No \\
\hline 60 & 2 & 3 & Sirit & No \\
\hline 61 & 1 & 2 & Alien & Yes \\
\hline 62 & 1 & 2 & Alien & No \\
\hline 63 & 1 & 2 & Alien & No \\
\hline 64 & 1 & 2 & Alien & Yes \\
\hline 65 & 1 & 2 & Alien & Yes \\
\hline 66 & 1 & 2 & Alien & No \\
\hline 67 & 1 & 2 & Alien & Yes \\
\hline 68 & 1 & 2 & Alien & Yes \\
\hline 69 & 1 & 2 & Alien & No \\
\hline 70 & 1 & 2 & Alien & No \\
\hline 71 & 1 & 1 & Alien & Yes \\
\hline 72 & 1 & 1 & Alien & No \\
\hline 73 & 1 & 1 & Alien & No \\
\hline 74 & 1 & 1 & Alien & Yes \\
\hline 75 & 1 & 1 & Alien & Yes \\
\hline 76 & 1 & 1 & Alien & No \\
\hline 77 & 1 & 1 & Alien & Yes \\
\hline 78 & 1 & 1 & Alien & Yes \\
\hline 79 & 1 & 1 & Alien & No \\
\hline
\end{tabular}




\begin{tabular}{|c|c|c|c|c|}
\hline 80 & 1 & 1 & Alien & No \\
\hline 81 & 1 & 2 & Sirit & Yes \\
\hline 82 & 1 & 2 & Sirit & No \\
\hline 83 & 1 & 2 & Sirit & No \\
\hline 84 & 1 & 2 & Sirit & Yes \\
\hline 85 & 1 & 2 & Sirit & Yes \\
\hline 86 & 1 & 2 & Sirit & No \\
\hline 87 & 1 & 2 & Sirit & Yes \\
\hline 88 & 1 & 2 & Sirit & Yes \\
\hline 89 & 1 & 2 & Sirit & No \\
\hline 90 & 1 & 2 & Sirit & No \\
\hline 91 & 2 & 1 & Sirit & Yes \\
\hline 92 & 2 & 1 & Sirit & No \\
\hline 93 & 2 & 1 & Sirit & No \\
\hline 94 & 2 & 1 & Sirit & Yes \\
\hline 95 & 2 & 1 & Sirit & Yes \\
\hline 96 & 2 & 1 & Sirit & No \\
\hline 97 & 2 & 1 & Sirit & Yes \\
\hline 98 & 2 & 1 & Sirit & Yes \\
\hline 99 & 2 & 1 & Sirit & No \\
\hline 100 & 2 & 1 & Sirit & No \\
\hline 101 & 2 & 2 & Alien & Yes \\
\hline 102 & 2 & 2 & Alien & No \\
\hline 103 & 2 & 2 & Alien & No \\
\hline 104 & 2 & 2 & Alien & Yes \\
\hline 105 & 2 & 2 & Alien & Yes \\
\hline 106 & 2 & 2 & Alien & No \\
\hline 107 & 2 & 2 & Alien & Yes \\
\hline 108 & 2 & 2 & Alien & Yes \\
\hline 109 & 2 & 2 & Alien & No \\
\hline 110 & 2 & 2 & Alien & No \\
\hline 111 & 1 & 4 & Sirit & Yes \\
\hline 112 & 1 & 4 & Sirit & No \\
\hline 113 & 1 & 4 & Sirit & No \\
\hline 114 & 1 & 4 & Sirit & Yes \\
\hline 115 & 1 & 4 & Sirit & Yes \\
\hline 116 & 1 & 4 & Sirit & No \\
\hline 117 & 1 & 4 & Sirit & Yes \\
\hline 118 & 1 & 4 & Sirit & Yes \\
\hline 119 & 1 & 4 & Sirit & No \\
\hline 120 & 1 & 4 & Sirit & No \\
\hline 121 & 1 & 3 & Sirit & No \\
\hline
\end{tabular}




\begin{tabular}{|c|c|c|c|c|}
\hline 122 & 1 & 3 & Sirit & Yes \\
\hline 123 & 1 & 3 & Sirit & Yes \\
\hline 124 & 1 & 3 & Sirit & No \\
\hline 125 & 1 & 3 & Sirit & No \\
\hline 126 & 1 & 3 & Sirit & Yes \\
\hline 127 & 1 & 3 & Sirit & No \\
\hline 128 & 1 & 3 & Sirit & Yes \\
\hline 129 & 1 & 3 & Sirit & No \\
\hline 130 & 1 & 3 & Sirit & Yes \\
\hline 131 & 2 & 4 & Alien & No \\
\hline 132 & 2 & 4 & Alien & Yes \\
\hline 133 & 2 & 4 & Alien & Yes \\
\hline 134 & 2 & 4 & Alien & No \\
\hline 135 & 2 & 4 & Alien & No \\
\hline 136 & 2 & 4 & Alien & No \\
\hline 137 & 2 & 4 & Alien & Yes \\
\hline 138 & 2 & 4 & Alien & No \\
\hline 139 & 2 & 4 & Alien & Yes \\
\hline 140 & 2 & 4 & Alien & Yes \\
\hline 141 & 2 & 3 & Alien & No \\
\hline 142 & 2 & 3 & Alien & Yes \\
\hline 143 & 2 & 3 & Alien & Yes \\
\hline 144 & 2 & 3 & Alien & No \\
\hline 145 & 2 & 3 & Alien & Yes \\
\hline 146 & 2 & 3 & Alien & No \\
\hline 147 & 2 & 3 & Alien & Yes \\
\hline 148 & 2 & 3 & Alien & No \\
\hline 149 & 2 & 3 & Alien & No \\
\hline 150 & 2 & 3 & Alien & Yes \\
\hline 151 & 1 & 1 & Sirit & No \\
\hline 152 & 1 & 1 & Sirit & Yes \\
\hline 153 & 1 & 1 & Sirit & Yes \\
\hline 154 & 1 & 1 & Sirit & No \\
\hline 155 & 1 & 1 & Sirit & No \\
\hline 156 & 1 & 1 & Sirit & Yes \\
\hline 157 & 1 & 1 & Sirit & No \\
\hline 158 & 1 & 1 & Sirit & Yes \\
\hline 159 & 1 & 1 & Sirit & No \\
\hline 160 & 1 & 1 & Sirit & Yes \\
\hline 161 & 2 & $2 Z$ & Sirit & Yes \\
\hline 162 & 2 & $2 Z$ & Sirit & No \\
\hline 163 & 2 & $2 Z$ & Sirit & Yes \\
\hline
\end{tabular}




\begin{tabular}{|c|c|c|c|c|}
\hline 164 & 2 & $2 Z$ & Sirit & Yes \\
\hline 165 & 2 & $2 Z$ & Sirit & No \\
\hline 166 & 2 & $2 Z$ & Sirit & Yes \\
\hline 167 & 2 & $2 Z$ & Sirit & No \\
\hline 168 & 2 & $2 Z$ & Sirit & No \\
\hline 169 & 2 & $2 Z$ & Sirit & No \\
\hline 170 & 2 & $2 Z$ & Sirit & Yes \\
\hline 171 & 1 & $2 Z$ & Alien & Yes \\
\hline 172 & 1 & $2 Z$ & Alien & Yes \\
\hline 173 & 1 & $2 Z$ & Alien & No \\
\hline 174 & 1 & $2 Z$ & Alien & Yes \\
\hline 175 & 1 & $2 Z$ & Alien & No \\
\hline 176 & 1 & $2 Z$ & Alien & No \\
\hline 177 & 1 & $2 Z$ & Alien & No \\
\hline 178 & 1 & $2 Z$ & Alien & No \\
\hline 179 & 1 & $2 Z$ & Alien & Yes \\
\hline 180 & 1 & $2 Z$ & Alien & Yes \\
\hline 181 & 1 & $2 Z$ & Sirit & Yes \\
\hline 182 & 1 & $2 Z$ & Sirit & No \\
\hline 183 & 1 & $2 Z$ & Sirit & No \\
\hline 184 & 1 & $2 Z$ & Sirit & No \\
\hline 185 & 1 & $2 Z$ & Sirit & No \\
\hline 186 & 1 & $2 Z$ & Sirit & No \\
\hline 187 & 1 & $2 Z$ & Sirit & Yes \\
\hline 188 & 1 & $2 Z$ & Sirit & Yes \\
\hline 189 & 1 & $2 Z$ & Sirit & Yes \\
\hline 190 & 1 & $2 Z$ & Sirit & Yes \\
\hline 191 & 2 & $2 Z$ & Alien & No \\
\hline 192 & 2 & $2 Z$ & Alien & No \\
\hline 193 & 2 & $2 Z$ & Alien & Yes \\
\hline 194 & 2 & $2 Z$ & Alien & Yes \\
\hline 195 & 2 & $2 Z$ & Alien & Yes \\
\hline 196 & 2 & $2 Z$ & Alien & No \\
\hline 197 & 2 & $2 Z$ & Alien & No \\
\hline 198 & 2 & $2 Z$ & Alien & Yes \\
\hline 199 & 2 & $2 Z$ & Alien & No \\
\hline 200 & 2 & $2 Z$ & Alien & Yes \\
\hline
\end{tabular}


Table 10: Variables of Interest

\begin{tabular}{|c|c|c|}
\hline $\begin{array}{c}\text { Independent } \\
\text { Variables }\end{array}$ & Description & Levels \\
\hline Door Type & A double or single doorway & 2 \\
Antenna & $\begin{array}{c}\text { One antenna at top of door frame, Two antennas } \\
\text { on the sides, Two antennas with one on top of door } \\
\text { frame, antennas both on top and sides, or four } \\
\text { Placement }\end{array}$ & 5 \\
Reader brand & Alien or Sirit readers & 2 \\
Metallic doorway & Presence of metal in door frame & 2 \\
Tag Type & Three tag models & 3 \\
Tag Orientation & Tags positioned in the x, y, and $\mathrm{z}$ axis & 3 \\
\hline
\end{tabular}

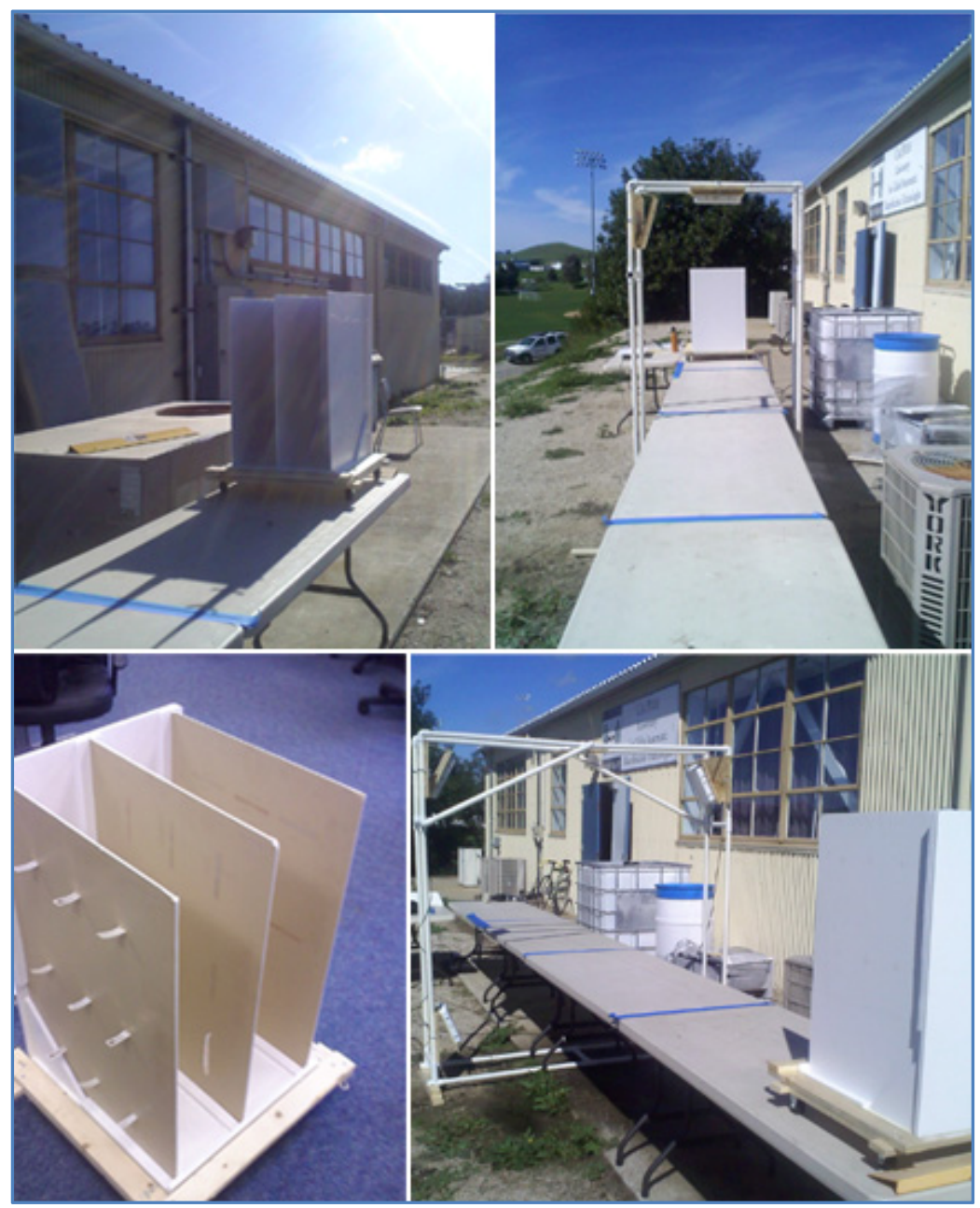

Figure 21: Actual Experiment 


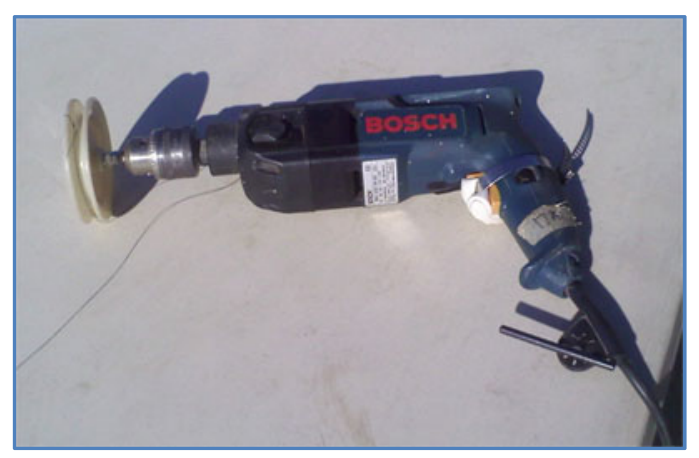

Figure 22: Drill used to maintain constant speed

Table 11: Ordinal Logistic Regression output Minitab (no interactions)

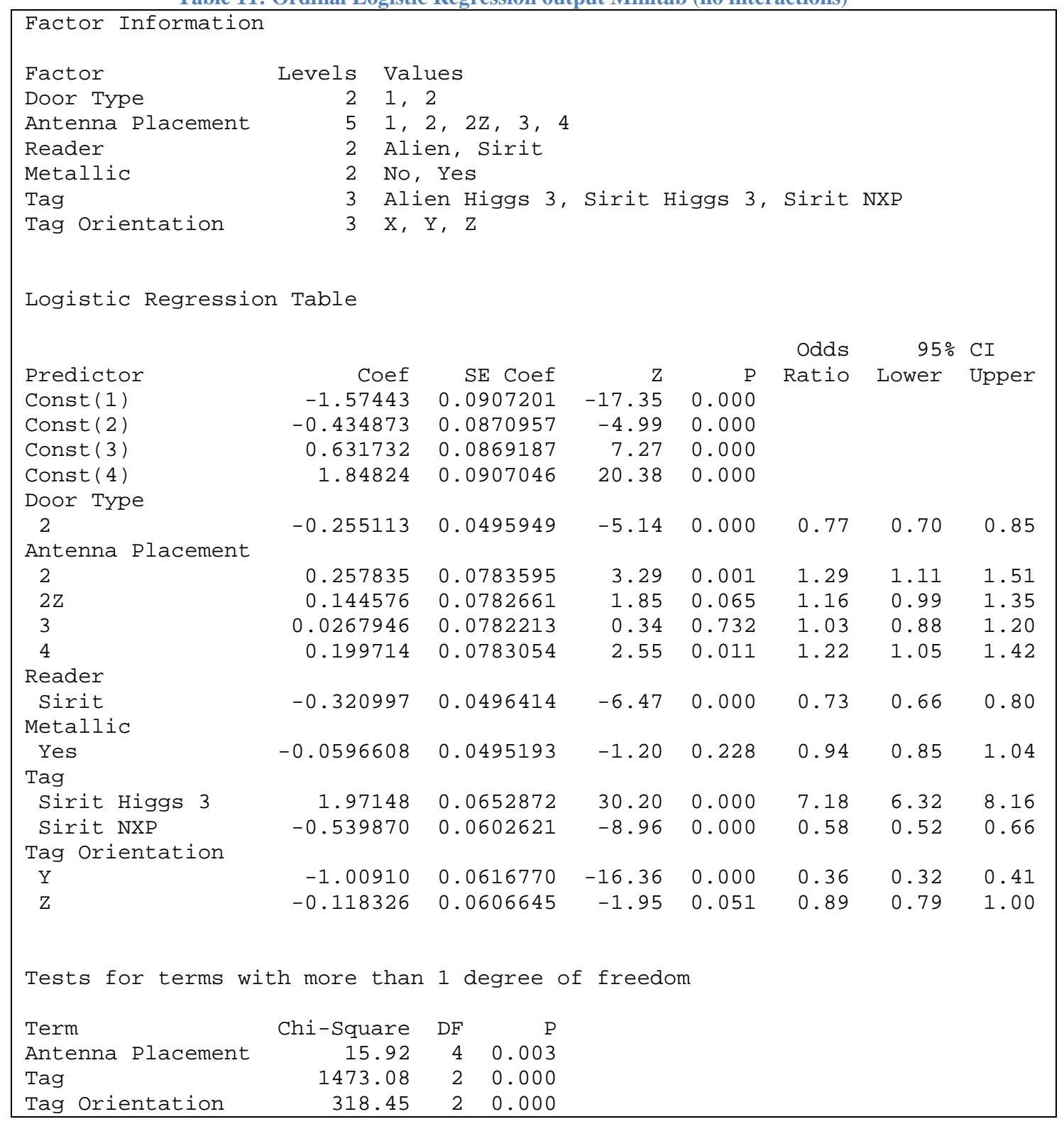


Log-Likelihood $=-7767.025$

Test that all slopes are zero: $G=1826.664, \mathrm{DF}=11, \mathrm{P}$-Value $=0.000$

Table 12: Ordinal Logistic Regression (no interactions)

The SAS System 13:26 Tuesday, March 23, 20101252

The LOGISTIC Procedure

Model Information

$\begin{array}{ll}\text { Data Set } & \text { WORK.ANALYSIS } \\ \text { Response Variable } & \text { Ordinal } \\ \text { Number of Response Levels } & 5 \\ \text { Model } & \text { cumulative logit } \\ \text { Optimization Technique } & \text { Fisher's scoring }\end{array}$

Number of Observations Read $\quad 5400$

Number of Observations Used $\quad 5400$

Response Profile

Ordered Total

Value Ordinal Frequency

$\begin{array}{rrr}1 & \text { E } & 1055 \\ 2 & \text { D } & 1083 \\ 3 & \text { C } & 1058 \\ 4 & \text { B } & 999 \\ 5 & \text { A } & 1205\end{array}$

Probabilities modeled are cumulated over the lower Ordered Values.

Class Level Information

Class

Door_Type

Antenna_Placement

1

$2 Z$

3

Reader

Metallic

Tag

The SAS System

4

Value

10

$\begin{array}{ll}1 & 0\end{array}$

0

1

Alien -0

Sirit 1

No $\quad 0$

Yes 1

Alien $\mathrm{Hi} \quad 0 \quad 0$

Sirit $\mathrm{Hi} \quad 1 \quad 0$

Sirit NX $\quad 0 \quad 1$

$\begin{array}{lll}x & 0\end{array}$

13:26 Tuesday, March 23, 20101253

The LOGISTIC Procedure

Class Level Information 


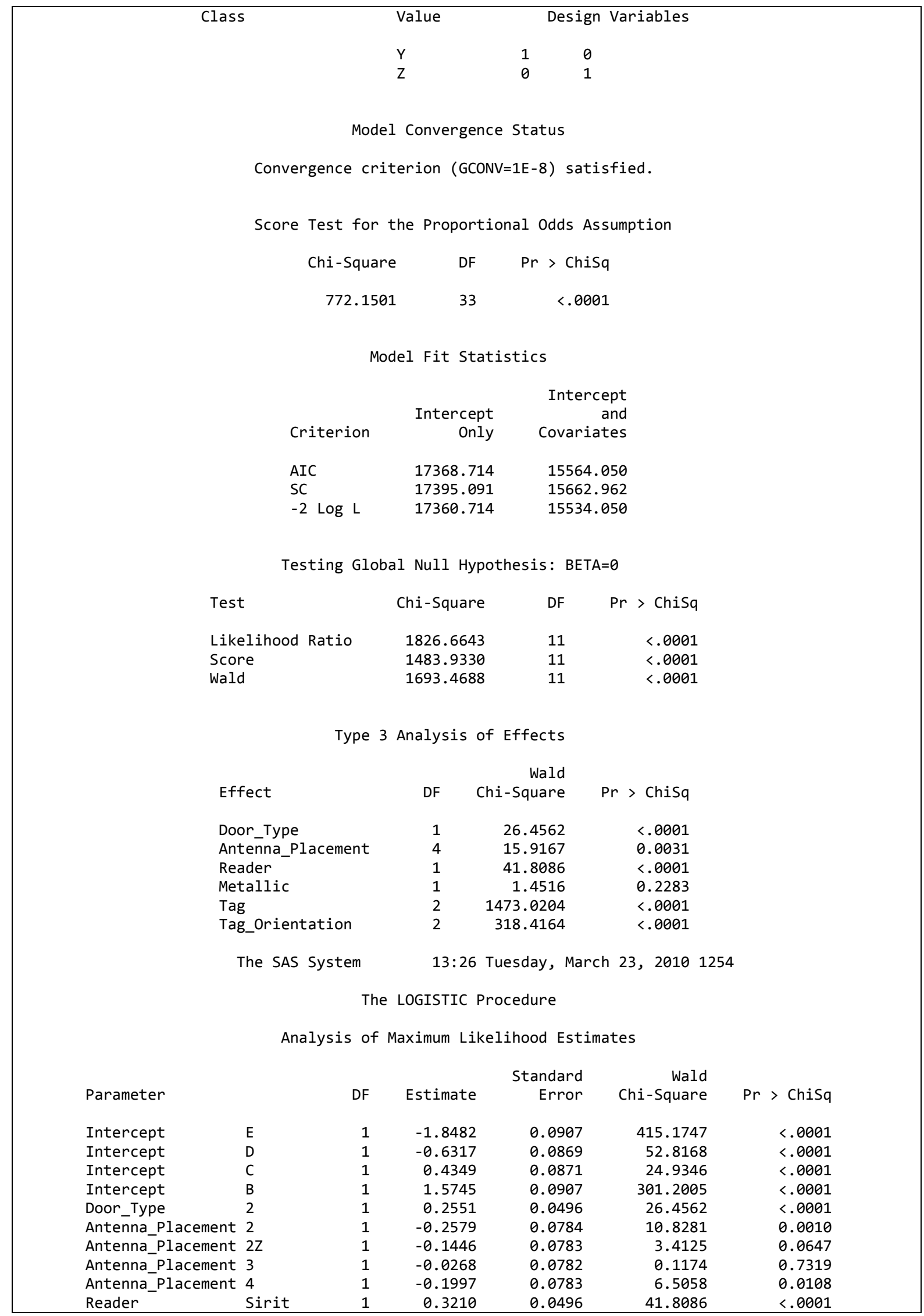




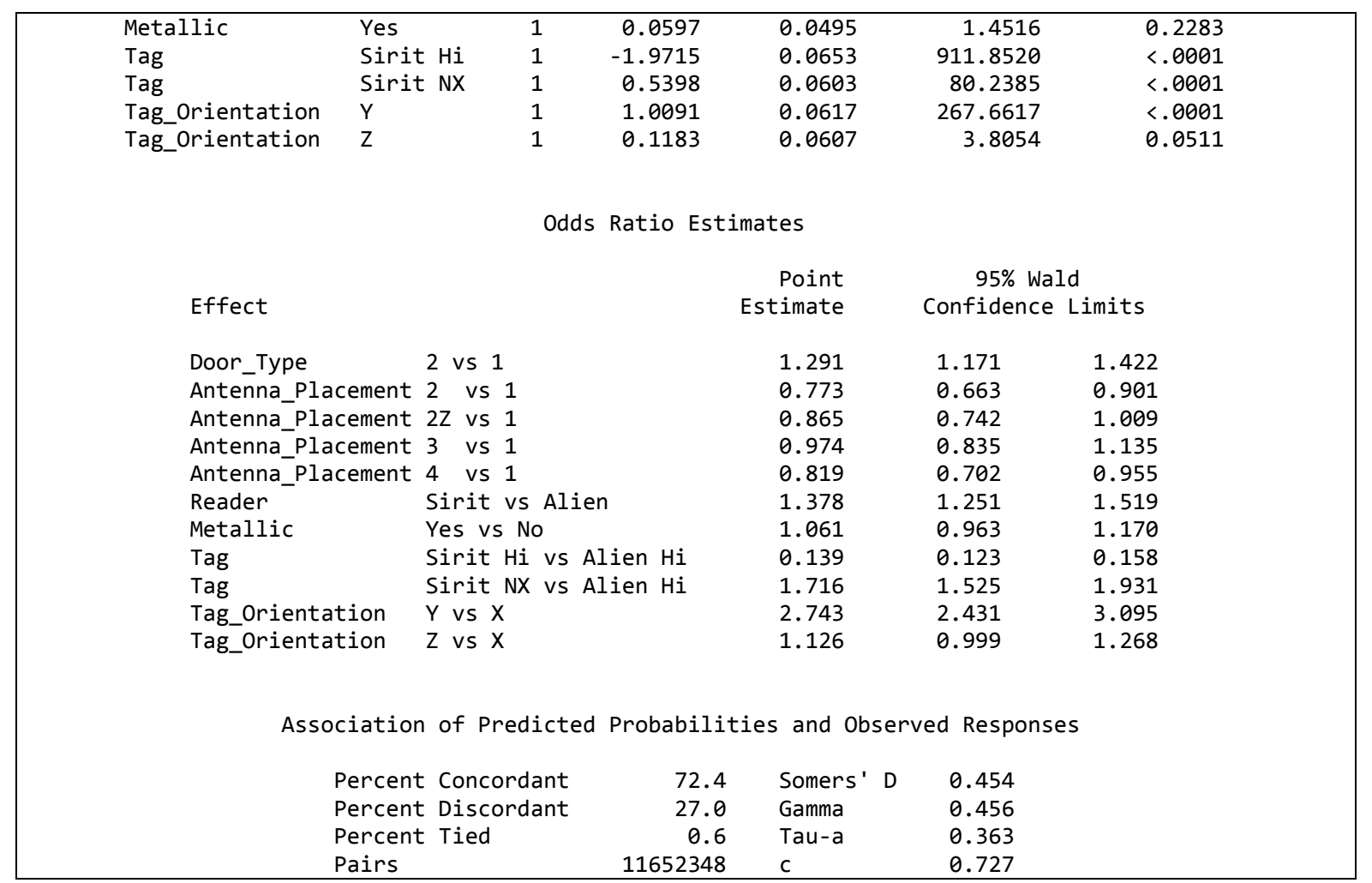

Table 13: Ordinal Logistic Regression (8 interactions)

The SAS System 10:47 Wednesday, March 24, 20101900

The LOGISTIC Procedure

Model Information

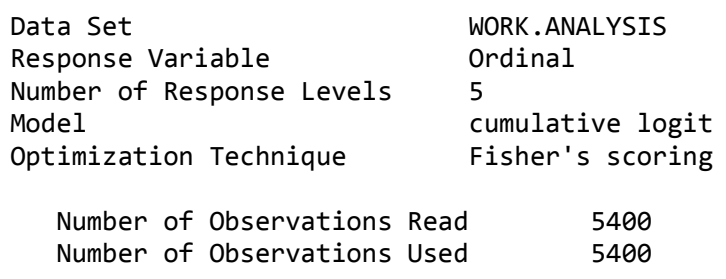

Response Profile

$\begin{array}{rlr}\begin{array}{r}\text { Ordered } \\ \text { Value }\end{array} & \text { Ordinal } & \begin{array}{r}\text { Total } \\ \text { Frequency }\end{array} \\ 1 & \text { E } & 1055 \\ 2 & \text { D } & 1083 \\ 3 & \text { C } & 1058 \\ 4 & \text { B } & 999 \\ 5 & \text { A } & 1205\end{array}$

Probabilities modeled are cumulated over the lower Ordered Values.

Class Level Information 


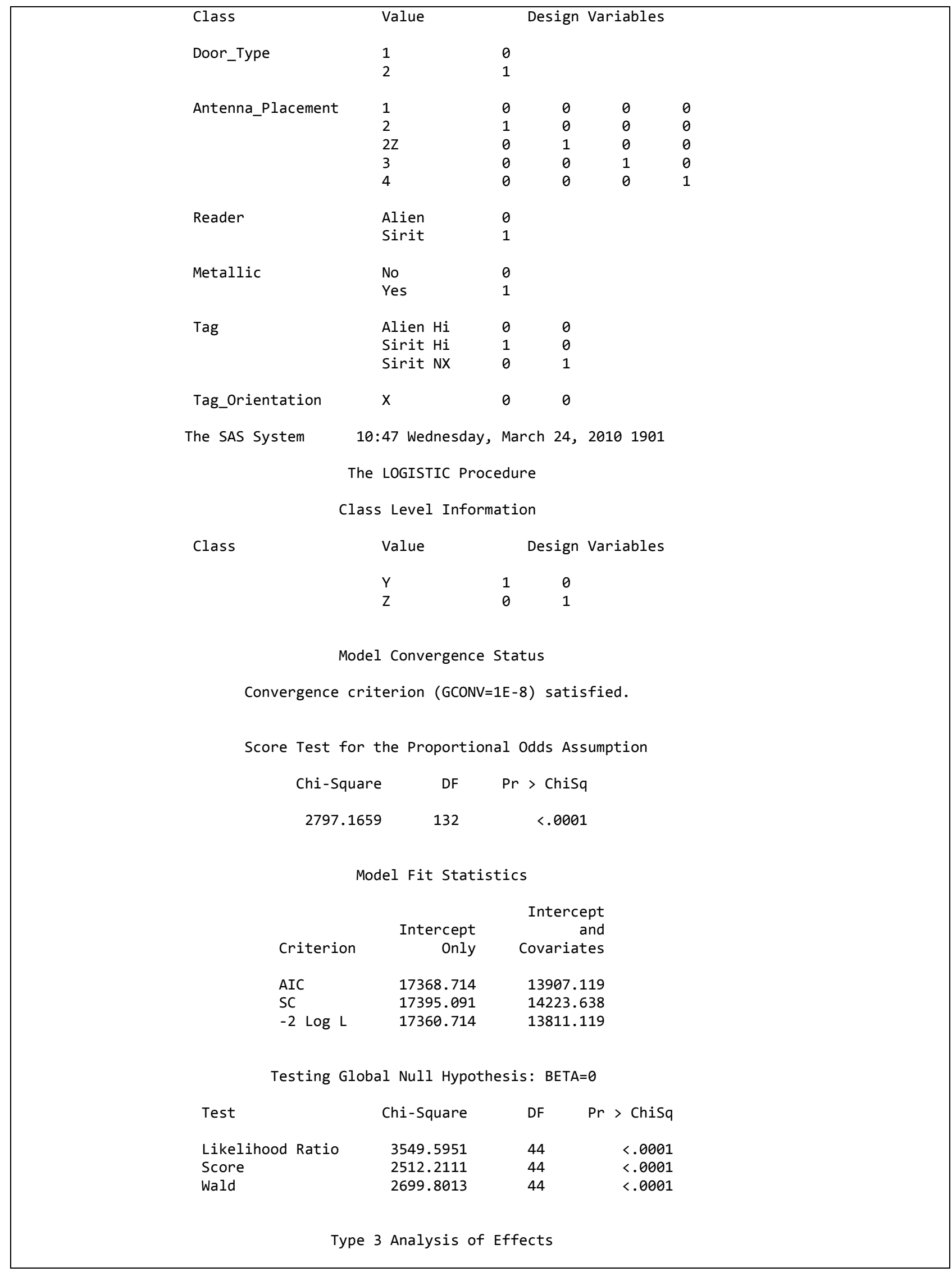




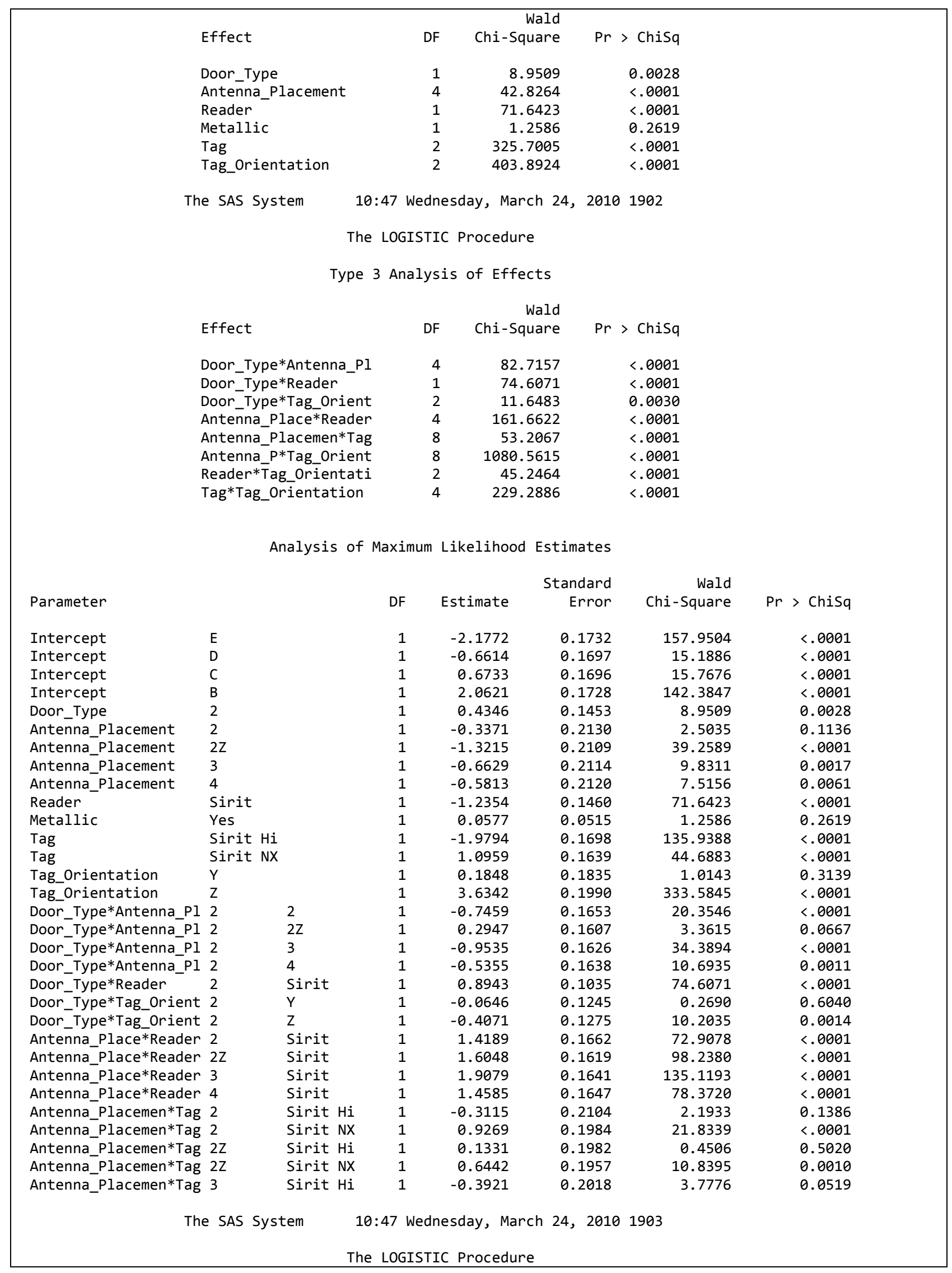




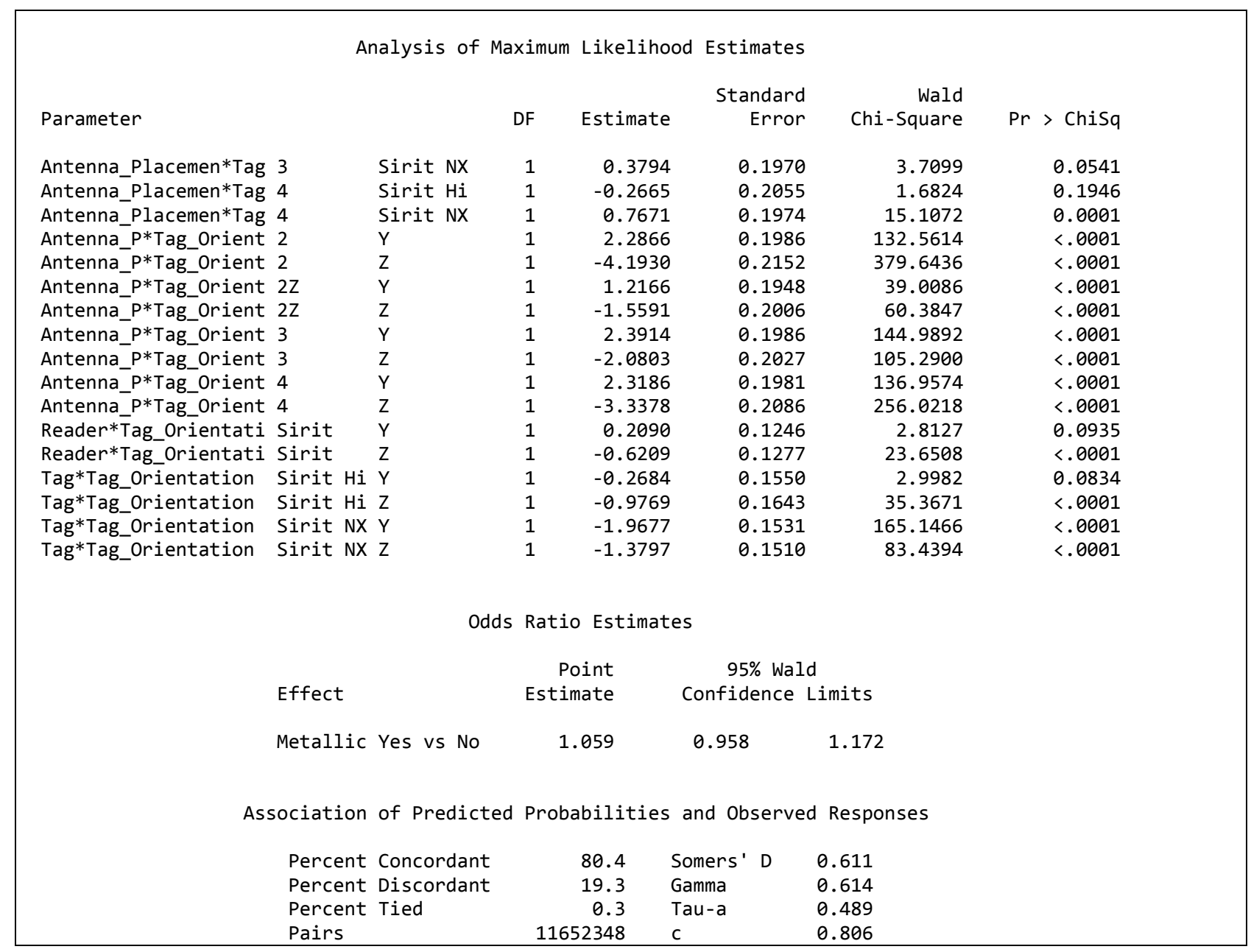

Table 14: Ordinal Logistic Regression (Final Model)

The SAS System 13:47 Wednesday, March 24, 20101

The LOGISTIC Procedure

Model Information

Data Set

WORK. ANALYSIS

Response Variable

Ordinal

Number of Response Levels Model

5

Optimization Technique

cumulative logit

Fisher's scoring

Number of Observations Read 5400

Number of Observations Used 5400

Response Profile

$\begin{array}{rlr}\begin{array}{r}\text { Ordered } \\ \text { Value }\end{array} & \text { Ordinal } & \begin{array}{r}\text { Total } \\ \text { Frequency }\end{array} \\ 1 & \text { E } & 1055 \\ 2 & \text { D } & 1083 \\ 3 & \text { C } & 1058 \\ 4 & \text { B } & 999 \\ 5 & \text { A } & 1205\end{array}$


Probabilities modeled are cumulated over the lower Ordered Values.

Class Level Information

Class

Value

Design Variables

Door_Type

1

0

21

Antenna_Placement

1

$2 Z$

3
4

1

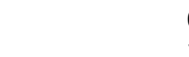

$\begin{array}{llll}0 & 0 & 0 & 0\end{array}$

$\begin{array}{llll}1 & 0 & 0 & 0 \\ 0 & 1 & 0 & 0\end{array}$

$\begin{array}{llll}0 & 1 & 0 & 0\end{array}$

$\begin{array}{llll}0 & 0 & 1 & 0 \\ 0 & 0 & 0 & 1\end{array}$

Reader

Alien $\quad 0$

Metallic

Sirit 1

Metallic

No $\quad 0$

Tag

Yes

1

Alien $\mathrm{Hi} \quad 0 \quad 0$

Sirit $\mathrm{Hi} \quad 1 \quad 0$

Sirit NX $\quad 0 \quad 1$

Tag_Orientation

$x$

$0 \quad 0$

The SAS System

13:47 Wednesday, March 24, 20102

The LOGISTIC Procedure

Class Level Information

Class

Value

Design Variables

$\begin{array}{lll}Y & 1 & 0 \\ Z & 0 & 1\end{array}$

Model Convergence Status

Convergence criterion ( $G \mathrm{CONV}=1 \mathrm{E}-8$ ) satisfied.

Score Test for the Proportional Odds Assumption

$\begin{array}{rrr}\text { Chi-Square } & \text { DF } & \text { Pr }>\text { ChiSq } \\ 2001.6459 & 81 & <.0001\end{array}$

Model Fit Statistics

$\begin{array}{lrr}\text { Criterion } & \begin{array}{r}\text { Intercept } \\ \text { Only }\end{array} & \begin{array}{r}\text { Intercept } \\ \text { and } \\ \text { Covariates }\end{array} \\ \text { AIC } & & \\ \text { SC } & 17368.714 & 14142.614 \\ \text {-2 Log L } & 17395.091 & 14347.033 \\ & 17360.714 & 14080.614\end{array}$

Testing Global Null Hypothesis: BETA=0

Test Chi-Square DF Pr $>$ ChiSq




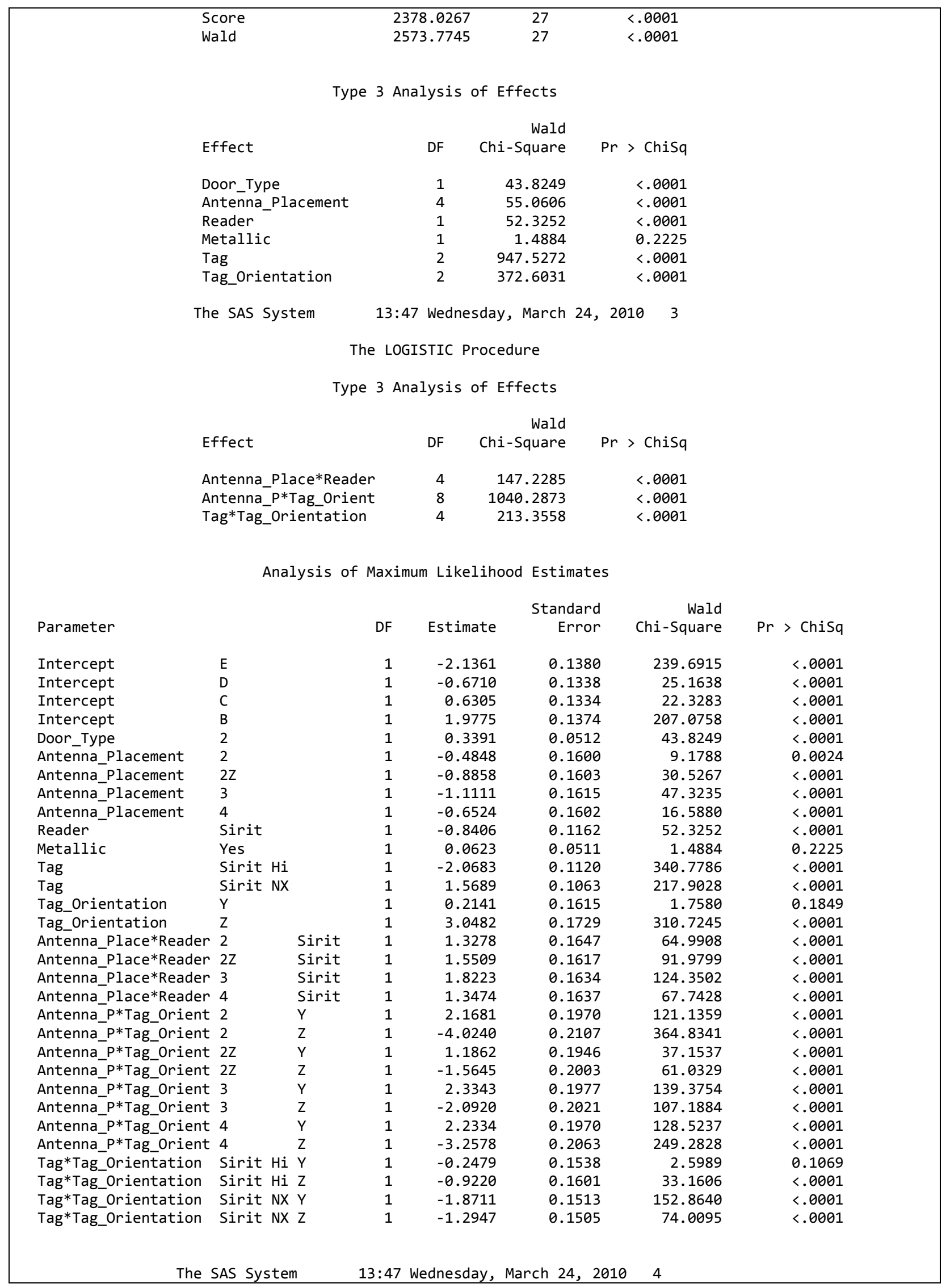


The LOGISTIC Procedure

Odds Ratio Estimates

Effect

Door_Type 2 vs 1

Metallic Yes vs No
Point

Estimate

$\begin{array}{lll}1.404 & 1.270 & 1.552 \\ 1.064 & 0.963 & 1.176\end{array}$

Association of Predicted Probabilities and Observed Responses

$\begin{array}{lrll}\text { Percent Concordant } & 79.4 & \text { Somers' D } & 0.592 \\ \text { Percent Discordant } & 20.2 & \text { Gamma } & 0.594 \\ \text { Percent Tied } & 0.3 & \text { Tau-a } & 0.473 \\ \text { Pairs } & 11652348 & \text { C } & 0.796\end{array}$

filename data DDE 'Excel|E: \SAS\[AllData.xls]data!r2c1:r5401c11' notab; libname Thesis 'E: \SAS';

Data Analysis;

infile data dsd missover notab dlm='09'x lrecl=2000;

Input

Day \$

Run

Door_type

Antenna_Placement \$

Reader \$

Metallic \$

Tag $\$$

Tag_ID \$

Tag_orientation \$

Data_Count

Ordinal \$;

run;

proc logistic data=Analysis descending;

class Day (reference="A") / param=ref;

model Ordinal = Day / link=clogit aggregate;

output out=probs predicted=prob;

run;

proc logistic data=Analysis descending;

class Door_type (reference="1") Antenna_Placement (reference="1") Reader (reference="Alien") Metallic (reference="No") Tag (reference="Alien Hi")

Tag_orientation (reference="X")/ param=ref;

model Ordinal = Door_Type Antenna_Placement Reader Metallic Tag

Tag_Orientation / link=clogit aggregate;

output out=probs predicted=prob;

run;

proc logistic data=Analysis descending;

class Door_Type (reference="1") Antenna_Placement (reference="1") Reader

(reference="Alien") Metallic (reference="No") Tag (reference="Alien Hi")

Tag_Orientation (reference="X") / param=ref;

/*model Ordinal = Door_Type Antenna_Placement Reader Metallic Tag 


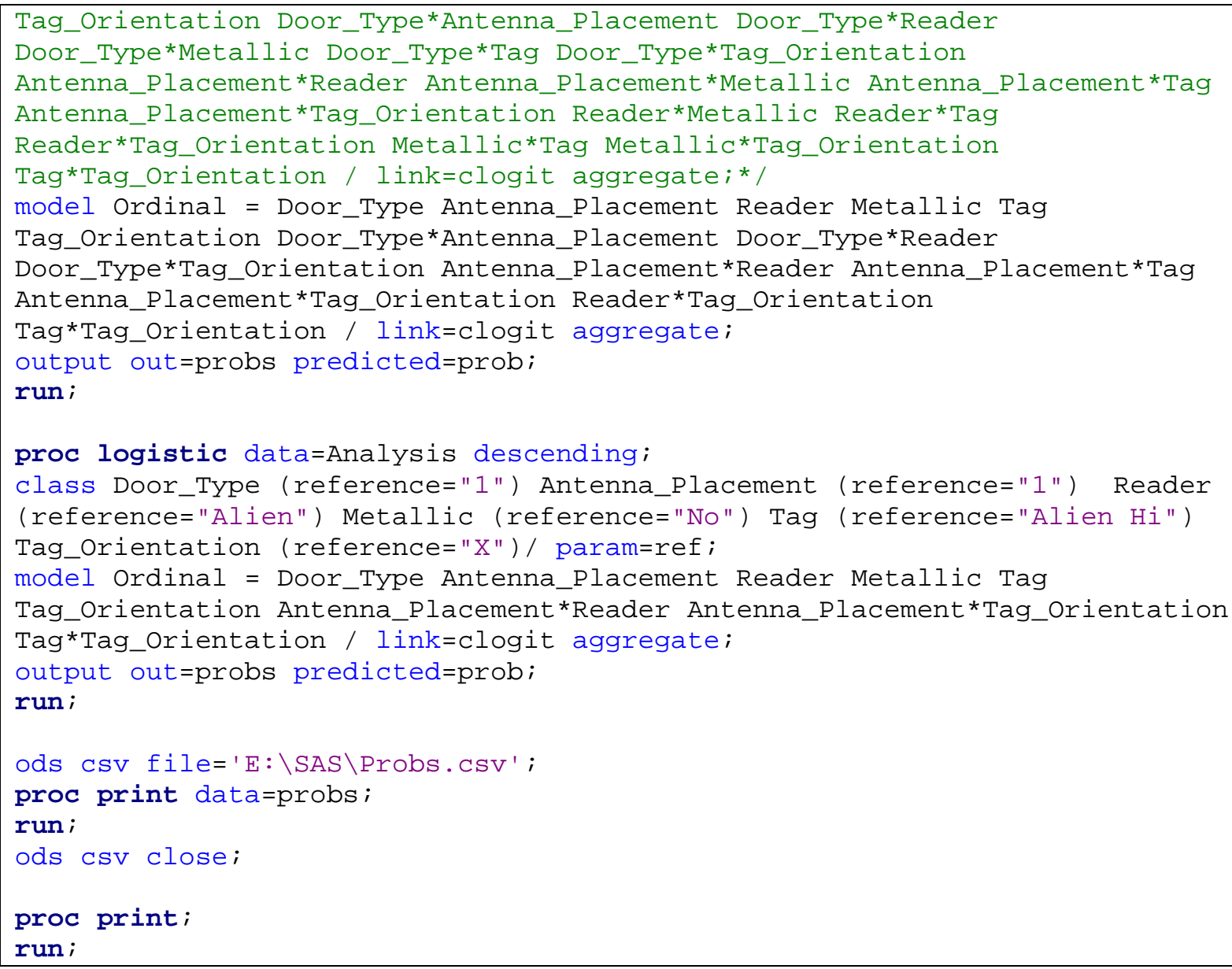




\section{Bibliography}

Agresti, Alan. "An Introduction to Categorical Data Analysis." (2007).

Aroor, Supreetha Rao and Daniel D. Deavours. "Evaluation of the State of

Passive UHF RFID: An Experimental Approach." (2007).

Canaris, David. Email. 2/15/2010.

Clarke, Robert H., et al. "Radio Frequency Identification (RFID) Performance:

The Effect of Tag Orientation and Package Contents." 19 (2006): 45-54.

Derbek, V., et al. "A UHF RFID measurement and evaluation test system."

Elektrotech. Inf.tech. 124.11 (2007): 384-390.

Dobkin, Daniel M. and Steven M. Weigand. "Evironmental Effects on RFID Tag Antennas." IEEE International Microwave Symposium. 2005.

Dobkin, Daniel M. RFID Basics: Antenna Polarization. 2008. 022010 <www.rfidtribe.org>.

Hodges, Steve, et al. "Assessing and Optimizing the Range of UHF RFID to Enable Real-World Pervasive Computing Applications." Proceedings of International Conference on Pervasive Computing. 2007.

Ramakrishnan, Karthik Moncombu and Daniel D. Deavours. "Performance Benchmarks for Passive UHF RFID Tags." Proceedings of the 13th GI/ITG. 2006.

RFID System Components and Costs. 2005. 022010

$\langle$ http://www.rfidjournal.com>.

Sarma, Sanjay E. and Daniel W. Engels. On the Future of RFID Tags and Protocols. White paper. Auto-ID Center, MIT, 2003.

Singh, Jay, et al. "RFID Tag Readability Issues with Palletized Loads of Consumer Goods." Packaging Technology and Science (2009): 431-441.

Sweeney, Patrick J. II. RFID For Dummies. Indianapolis, Indiana: Wiley Publishing, Inc., 2005.

Weigand, Steven M. and Daniel M. Dobkin. "Multiple RFID Tag Plane Array Effects." Antennas and Propagation Society International Symposium, 2006.

Zou, Wen bin, Yun Wu and Yong Zhao. "Automatic Testing System for UHF Passive RFID Tag Performance." International Conference on Networking and Digital Society. 2009. 79-82. 